\title{
Violation of the FRW consistency condition as a signature of backreaction
}

\section{Boehm, Celine}

2013-09

Boehm , C \& Räsänen , S 2013, ' Violation of the FRW consistency condition as a signature of backreaction ' , Journal of Cosmology and Astroparticle Physics , vol. 2013 , no. 9 , 003 . https://doi.org/10.1088/1

http://hdl.handle.net/10138/310424

https://doi.org/10.1088/1475-7516/2013/09/003

other

acceptedVersion

Downloaded from Helda, University of Helsinki institutional repository.

This is an electronic reprint of the original article.

This reprint may differ from the original in pagination and typographic detail.

Please cite the original version. 


\title{
Violation of the FRW consistency condition as a signature of backreaction
}

\author{
Céline Boehm ${ }^{a}$ and Syksy Räsänen ${ }^{b}$ \\ ${ }^{a}$ Institute for Particle Physics Phenomenology, Durham University \\ South Road, Durham, DH1 3LE, United Kingdom \\ and LAPTH, U. de Savoie, CNRS \\ BP 110, 74941 Annecy-Le-Vieux, France \\ ${ }^{b}$ University of Helsinki, Department of Physics \\ and Helsinki Institute of Physics \\ P.O. Box 64, FIN-00014 University of Helsinki, Finland \\ E-mail: c $d o t \mathrm{~m} d o t$ boehm at durham dot ac dot uk, \\ syksy dot rasanen at iki $\operatorname{dot} \mathrm{fi}$
}

\begin{abstract}
We propose a backreaction toy model with realistic features and confront it with the Union2.1 supernova data. The model provides a good fit even though the expansion history is quite different from the $\Lambda \mathrm{CDM}$ model and the effective equation of state is far from -1 . We discuss compatibility (or lack thereof) with other observations. We show that the FRW consistency condition between distance and expansion rate is violated with an amplitude that is slightly below the current observational limits. We expect that this is also the case in the real universe if backreaction is significant, providing a distinct signature of backreaction.
\end{abstract}




\section{Contents}

1 Introduction 1

2 Backreaction toy model and datafit $\quad 2$

2.1 Backreaction toy model 2

2.2 Fit to Union2.1 data 5

3 Signatures of backreaction $\quad 7$

3.1 Deviation from FRW behaviour 7

$\begin{array}{lll}3.2 & \text { Low redshift expansion } & 12\end{array}$

$\begin{array}{lll}3.3 & \text { Other observations } & 14\end{array}$

4 Conclusion

\section{Introduction}

Observations. The observed average expansion rate and distance are at late times about a factor of two higher than predicted by the homogeneous and isotropic and spatially flat matter-dominated cosmological model (see [1] for discussion and references). There are three possible explanations: an exotic matter component with negative pressure, failure of general relativity on cosmological scales or the breakdown of the validity of the homogeneous and isotropic approximation.

Most observations are consistent with extending the matter-dominated spatially flat Friedmann-Robertson-Walker (FRW) model to the $\Lambda$ CDM model by simply adding vacuum energy. There are a few discrepancies, however. Temperature variations in the cosmic microwave background (CMB) along lines of sight that intersect density extrema of the largescale structure are much higher than expected [2], and there are also other CMB anomalies on large angular scales [3]. The distribution of galaxies on large scales appears to be less homogeneous than expected, particularly when it comes to extreme structures [4-6], though the overall amplitude of the two-point function is consistent with the $\Lambda$ CDM model [7].

It has also been argued that observations of supernovae (SNe), supplemented by other data, favour departures from the $\Lambda \mathrm{CDM}$ model in the form of more acceleration today [8], less acceleration (or even deceleration) today [9] or more deceleration at early times [10]. The results depend strongly on the assumed parametrisation of the cosmological model [11] and choice of light curve fitter [12]. None of these deviations are statistically significant [13], but the analyses show that large departures from the $\Lambda \mathrm{CDM}$ expansion history are allowed. Currently, SNe are the most detailed probe of the distance-redshift relation (from which the expansion rate is inferred), but observations of large-scale structure, in particular the baryon acoustic oscillation (BAO) pattern [14-21] (see also [22]), offer an increasingly accurate picture of distances and expansion rates, though the analysis is more model-dependent. Galaxy ages are a relatively new and important model-independent way to measure the expansion rate, with systematics that are not yet as well-studied as for more established probes [23-26]. 
Backreaction. One possibility is that the factor two failure in the predictions of the homogeneous and isotropic matter-dominated model is related to the known breakdown of homogeneity and isotropy due to structure formation, instead of new fundamental physics [27-31]. The average expansion rate is in general affected by inhomogeneity and anisotropy, even if the distribution of matter is statistically homogeneous and isotropic. The effect of deviations from homogeneity and/or isotropy on the average expansion rate is known as backreaction [32-36] (see [1, 37, 38] for reviews). Inhomogeneity can increase the average expansion rate, and it can even lead to accelerating expansion in a dust universe [39, 40]. In a statistically homogeneous and isotropic universe, faster expansion rate implies larger redshifts and distances [41, 42]. The possibility that change in the average expansion rate due to structure formation would explain the late-time observations of faster expansion and longer distances is called the backreaction conjecture.

Given the standard scenario of gravitational structure formation from small Gaussian perturbations in baryons and cold dark matter, there are no free parameters for backreaction. The initial matter and radiation densities and the perturbation spectrum is known modelindependently from CMB observations [43, 44]. Therefore, the average expansion rate is fully determined in principle. However, in practice it is difficult to calculate the effect of non-linear structures. In Newtonian cosmology [34] and in relativistic perturbation theory [45] (see also [46]), the effect is small, given certain assumptions. In a semirealistic model, backreaction has been found to be of the same order of magnitude as the observed change in the expansion rate, given certain approximations [47]. The magnitude of backreaction in the real universe is an open question.

However, backreaction can be tested by independent observations of distance and expansion rate, even without a prediction for the expansion rate. (If backreaction is significant, the expansion rate is not expected to be the same as in the $\Lambda \mathrm{CDM}$ model, so increasing agreement of observations with the $\Lambda \mathrm{CDM}$ model also tests backreaction indirectly.) In a FRW universe there is a specific relation between distance and expansion rate, which can be used to test the FRW metric [48]. If backreaction is significant, the distance-expansion rate relation is in general different from the FRW case [41, 42]. However, so far there has been no reliable estimate of the expected magnitude of the deviation in the real universe, nor of its redshift dependence. (A calculation with an ad hoc treatment of redshift and distance not based on light propagation equations was presented in [49]; see also [50].)

In this paper we consider a backreaction toy model where the expansion rate has features similar to those expected if backreaction is significant in the real universe. First, we show that the model fits the Union2.1 SN data [51] well, even though the expansion history is quite different from the $\Lambda \mathrm{CDM}$ case. Second, we quantify the violation of the FRW consistency condition between distance and expansion rate.

In section 2 we present the toy model, the results of the fit to the Union2.1 SN data and discuss the expansion history. In section 3 we consider effective equations of state, the deviation from the FRW relation between distance and expansion rate, and cosmological observations other than SNe. We conclude in section 4.

\section{Backreaction toy model and datafit}

\subsection{Backreaction toy model}

The expansion rate. In a statistically homogeneous and isotropic universe with a finite homogeneity scale, the average expansion rate describes the overall expansion of any region 
larger than the homogeneity scale. If the distribution of structures evolves slowly compared to the time it takes for light to cross the homogeneity scale, redshift and distance are expected to be calculable from the average expansion rate $[40-42,47,52]$. The homogeneity scale in the real universe appears to be of the order $100 \mathrm{Mpc}$, although it is debated whether this has been satisfactorily established observationally $[5,53-55]$. In any case, average quantities are not expected to give an accurate description on much smaller scales.

If backreaction increases the expansion rate significantly, this is related to large variance of the expansion rate due to increasing differentiation of overdense and underdense regions in the course of structure formation. We consider a simple toy model that allows for such evolution. The expansion rate is taken to be that of a union of two FRW dust models, one with positive and the other with negative spatial curvature. We take the dust energy density to be always non-negative. This is similar to the toy model studied in [40], but there the negatively curved model was taken to be completely empty. The universe is not considered to be physically composed of two regions, this is just a simple way to parametrise an expansion rate that in a realistic situation would arise from an average over a complicated distribution of structures. The positively curved part describes overdense regions, which slow down relative to the spatially flat case, and the negatively curved part represents underdense regions, which speed up relative to the spatially flat model.

The volume of the universe is proportional to $a^{3}=a_{1}^{3}+a_{2}^{3}$, where $a_{1}$ and $a_{2}$ are the scale factor of a negatively and positively curved FRW dust model, respectively. The average expansion rate is

$$
H \equiv \frac{\dot{a}}{a}=\frac{a_{1}^{3}}{a_{1}^{3}+a_{2}^{3}} H_{1}+\frac{a_{2}^{3}}{a_{1}^{3}+a_{2}^{3}} H_{2} \equiv v_{1} H_{1}+v_{2} H_{2},
$$

where dot stands for derivative with respect to time $t$ (physically, this is the proper time of observers comoving with the hypersurface of statistical homogeneity and isotropy [40-42, 47]) and $H_{i} \equiv \dot{a}_{i} / a_{i}$. The average energy density of a dust universe is $\left\langle\rho_{\mathrm{m}}\right\rangle \propto a^{-3}$.

The scale factors and the time $t$ are given in terms of the development angle $\phi$ and the evolution variable $\tilde{\phi}$ as

$$
\begin{array}{ll}
a_{1}=A_{1}(\cosh \tilde{\phi}-1), & t=T_{1}(\sinh \tilde{\phi}-\tilde{\phi}) \\
a_{2}=A_{2}(1-\cos \phi), & t=T_{2}(\phi-\sin \phi),
\end{array}
$$

where $A_{i}$ and $T_{i}$ are constants. The development angle varies from 0 to $2 \pi$ and $\tilde{\phi}$ runs from 0 to $\infty$. The big bang is at $\phi=0$. The function $\tilde{\phi}(\phi)$ is determined by equating $t$ in $(2.2)$ and (2.3). At early times, both regions behave like the Einstein-de Sitter (EdS) universe, the spatially flat matter-dominated FRW model. The underdense region decelerates less as time goes on, and asymptotically approaches the coasting empty universe. The overdense region slows down more over time, turns around from expansion to collapse at $\phi=\pi$ and collapses to a singularity at $\phi=2 \pi$. The toy model shows different behaviour for different parameter values. The universe can transition smoothly from early EdS behaviour towards a negatively curved FRW model, or there can be a period of extra deceleration followed by acceleration [40]. Note that acceleration may not be needed to explain the observations, because the relation between expansion rate and distance is different than in the FRW case [41, 42].

Physics depends on $A_{1}$ and $A_{2}$ only via the ratio $A_{1} / A_{2}$ and dimensionless quantities depend on $T_{1}$ and $T_{2}$ only via $T_{1} / T_{2}$. A large value of $A_{1} / A_{2}$ corresponds to large relative volume of the underdense region, and a large value of $T_{1} / T_{2}$ means that the underdense region 
evolves rapidly compared to the overdense region. It is more convenient to have parameters with a clearer physical interpretation and finite range. Therefore, instead of $A_{i}$ and $T_{i}$, we parametrise the model with the fraction of volume occupied by the overdense region in the beginning and at the time when it starts to collapse,

$$
\begin{aligned}
& f_{\mathrm{b}} \equiv v_{2}(\phi=0)=\frac{A_{2}^{3}}{A_{1}^{3}\left(T_{2} / T_{1}\right)^{2}+A_{2}^{3}} \\
& f_{\mathrm{c}} \equiv v_{2}(\phi=\pi)=\frac{8 A_{2}^{3}}{A_{1}^{3}[\cosh \tilde{\phi}(\pi)-1]^{3}+8 A_{2}^{3}} .
\end{aligned}
$$

The expansion history of the universe is determined by the constants $f_{\mathrm{b}}$ and $f_{\mathrm{c}}$, which have the ranges $0<f_{\mathrm{b}}<1,0<f_{\mathrm{c}}<\left[\frac{9 \pi^{2}}{16}\left(f_{\mathrm{b}}^{-1}-1\right)+1\right]^{-1}$. In addition, there is one parameter that determines at which point of the expansion history the universe is today. We take this to be the value of the development angle today, $\phi_{0}$ (the subscript 0 refers to quantities evaluated today). Were we to consider dimensional quantities, we would also have the value of the Hubble parameter today, $H_{0}$.

For comparison, in the $\Lambda$ CDM model, where the energy density consists of dust and vacuum energy, there is only one free parameter (we take the name $\Lambda$ CDM to imply spatial flatness), which determines at which point of the expansion history the universe is today. (Again excepting the overall scale, usually given by $H_{0}$.) We take this to be the value of the matter density parameter $\Omega_{\mathrm{m}} \equiv 8 \pi G_{\mathrm{N}} \rho_{\mathrm{m}} /\left(3 H^{2}\right)$ (where $G_{\mathrm{N}}$ is Newton's constant) today, so we have

$$
H=H_{0} \sqrt{1-\Omega_{\mathrm{m} 0}+\Omega_{\mathrm{m} 0} a^{-3}},
$$

where we have adopted the normalisation $a_{0}=1$. We also consider the OCDM model, which is the FRW model where the energy density consists of dust and the universe has negative spatial curvature. The expansion rate of the OCDM model is

$$
H=H_{0} \sqrt{\left(1-\Omega_{\mathrm{m} 0}\right) a^{-2}+\Omega_{\mathrm{m} 0} a^{-3}} .
$$

The redshift and the distance. We take the redshift $z$ to be given by [41, 42]

$$
1+z=a^{-1} .
$$

We assume that the angular diameter distance $D_{A}$ can be solved from $[41,42]$

$$
H \frac{\mathrm{d}}{\mathrm{d} z}\left[(1+z)^{2} H \frac{\mathrm{d} D_{A}}{\mathrm{~d} z}\right]=-4 \pi G_{\mathrm{N}}\left\langle\rho_{\mathrm{m}}\right\rangle D_{A}
$$

with the initial conditions $D_{A}(0)=0, \frac{\mathrm{d} D_{A}}{\mathrm{~d} z}(0)=H_{0}^{-1}$. In the FRW case, $\left\langle\rho_{\mathrm{m}}\right\rangle$ would be replaced by $\rho+p$, where $p$ is the pressure. Given $\left\langle\rho_{\mathrm{m}}\right\rangle \propto a^{-3}=(1+z)^{3},(2.8)$ can be written as

$$
h \frac{\mathrm{d}}{\mathrm{d} z}\left[(1+z)^{2} h \frac{\mathrm{d} d_{A}}{\mathrm{~d} z}\right]=-\frac{3}{2} \Omega_{\mathrm{m} 0}(1+z)^{3} d_{A},
$$

where we have introduced the notation $h \equiv H / H_{0}, d_{A} \equiv H_{0} D_{A}$; we also denote $d \equiv(1+z) d_{A}$. The distance is fully determined by the expansion history $h(z)$ and the value of $\Omega_{\mathrm{m} 0}$. The luminosity distance is $D_{L}=(1+z)^{2} D_{A}[56,57]$; we denote $d_{L} \equiv H_{0} D_{L}$. 


\begin{tabular}{|c|ccccccc|}
\hline Model & $\chi^{2}$ & $\chi^{2} /$ d.o.f. & $\Omega_{\mathrm{m} 0}$ & $q_{0}$ & $f_{\mathrm{b}}$ & $f_{\mathrm{c}}$ & $\phi_{0}$ \\
\hline \hline Model 1 & 548 & 0.94 & 0.35 & -0.52 & 0.995 & 0.215 & 6.24 \\
Model 2 & 551 & 0.95 & 0.28 & -0.39 & 0.974 & 0.168 & 5.99 \\
\hline$\Lambda$ CDM & 545 & 0.94 & 0.29 & -0.57 & - & - & - \\
OCDM & 566 & 0.98 & 0.00 & 0.00 & - & - & - \\
\hline
\end{tabular}

Table 1. Parameter values for backreaction models 1 and 2 and the best-fit $\Lambda$ CDM and OCDM models.

\subsection{Fit to Union2.1 data}

Supernova data fit. We fit the backreaction toy model defined by (2.1)-(2.4) to the Union2.1 SN data [51] using a Monte Carlo Markov Chain analysis. For comparison, we also fit the $\Lambda$ CDM model (2.5) and the OCDM model (2.6) to the same data. The OCDM model is included to illustrate how much backreaction improves the fit compared to the FRW dust case. The Union2.1 dataset consists of distances to 580 type Ia SNe between redshifts 0.015 and 1.4. Distances of the Union SNe are determined only relative to each other, the absolute distance scale is arbitrary, so the data only fixes $d_{L}$, not $D_{L}$.

The analysis leading to the publicly available Union2.1 distance data has been done assuming that the $\Lambda \mathrm{CDM}$ model is correct. As the SN light curve parameters and cosmological parameters have been fitted at the same time, the resulting distances are model-dependent, and may be biased towards the $\Lambda \mathrm{CDM}$ model. To properly consider other models, the data should be re-analysed from the beginning [58]. The results also depend on the choice of light curve fitter [12]. Furthermore, as the intrinsic dispersion of SN luminosity is adjusted to obtain a good fit with the $\Lambda \mathrm{CDM}$ model, absolute goodness-of-fit values are not meaningful. Nevertheless, relative goodness-of-fit statistics can be compared, bearing the above caveats in mind.

If the goal were to confront the result of a realistic calculation of the expansion rate due to backreaction with observations, it would be important to address the above issues. However, we are interested in seeing whether a toy model with realistic features can provide a reasonable fit to the data and in estimating the deviation of the distance-expansion rate relation from the FRW case, so the precise quality of the fit and the exact parameter values are not crucial. Likewise, the fact that the toy model has two more parameters than the $\Lambda \mathrm{CDM}$ model is not important.

Results of the data fit. We highlight two sets of parameter values, called models 1 and 2. Model 1 is the best-fitting model we found that satisfies the constraint $\Omega_{\mathrm{m} 0} \leq 0.35$. In order to demonstrate a different backreaction expansion history with a smaller matter density parameter, we also show model 2. The parameter values and $\chi^{2}$ numbers for models 1 and 2 , as well as for the best-fit $\Lambda$ CDM and OCDM models, are listed in table 1 . We also give the values of $\Omega_{\mathrm{m} 0}$ and $q_{0}$, where $q$ is the deceleration parameter, defined in (3.4).

The backreaction models are a significant improvement over the best-fit OCDM model (which is, as expected, the limit of a completely empty universe), and the quality of the fit is close to the $\Lambda \mathrm{CDM}$ model. The $\chi^{2} /$ d.o.f. is 0.94 for both the $\Lambda$ CDM model and model 1 , and 0.95 for model 2 (note that the data points are not independent). Taking into account the issues discussed above, the difference in $\chi^{2}$ is not large, $\Delta \chi^{2}=3$ for model 1 and $\Delta \chi^{2}=5$ for model 2. In both backreaction models, $f_{\mathrm{b}}$ is close to 1 , and it is possible that a more 


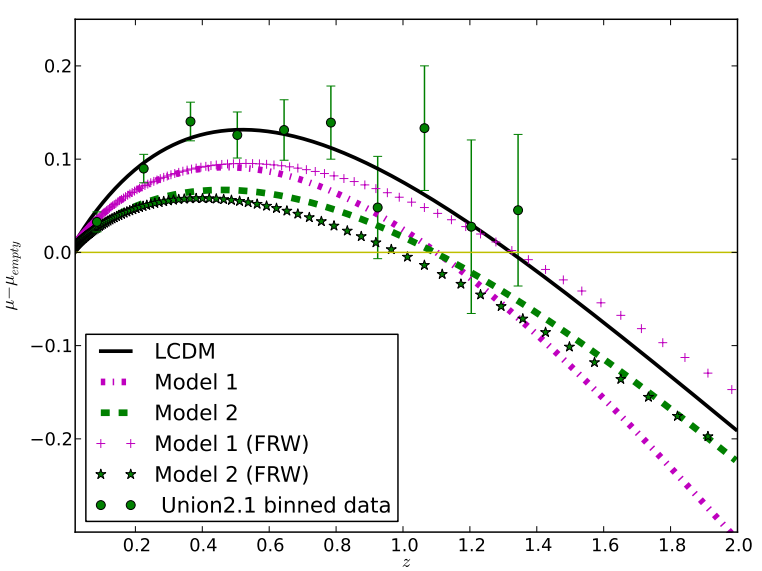

(a)

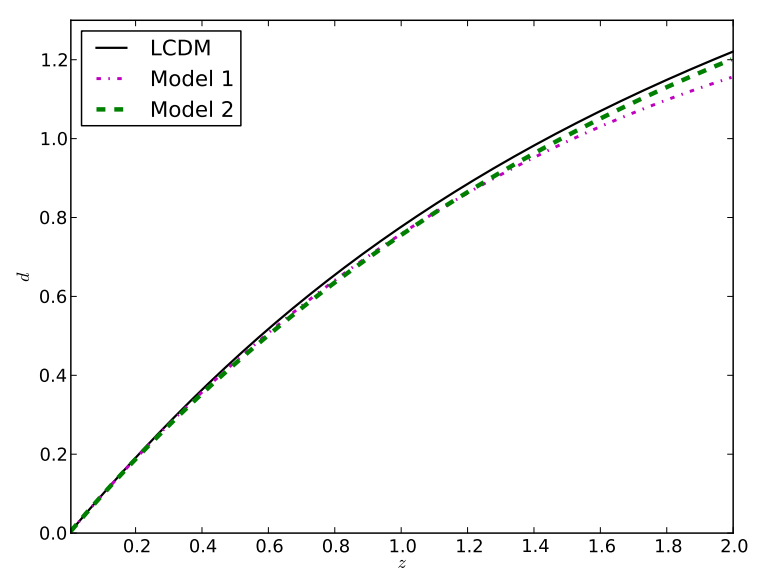

(b)

Figure 1. a) Magnitude relative to the empty FRW universe, corresponding to the parameters given in table 1. Dots indicate binned Union2.1 data. Crosses and stars mark the magnitude that models 1 and 2 would have were the relation between distance and expansion rate the same as in the FRW case; see section 3.1 . b) The distance $d=(1+z) d_{A}$.

detailed scan of the parameter space in the region around $f_{\mathrm{b}}=1$ would uncover models that fit the data better. Relaxing the constraint $\Omega_{\mathrm{m} 0} \leq 0.35$ does lead to better fitting models, with expansion histories even further away from the $\Lambda$ CDM model. As already mentioned, we are more interested in demonstrating qualitative features than in finding the optimal fit.

In figure $1 \mathrm{a}$ we show ${ }^{1}$ the magnitude-redshift relation in models 1 and 2 and in the $\Lambda$ CDM model, relative to the empty FRW universe, $\mu-\mu_{\text {empty }}=5 \log _{10}\left(d / d_{\text {empty }}\right)$. The corresponding distances are shown in figure $1 \mathrm{~b}$. The fact that the curves for the backreaction models are below the $\Lambda \mathrm{CDM}$ line at small $z$ reflects the fact that they accelerate less strongly today, but this is not a general feature. There are parameter values that give a good fit with more rapid acceleration today. In both model 1 and $2, \phi_{0}$ is not far from the maximum value $2 \pi$, indicating that the overdense region is rapidly collapsing and its fraction of the volume is small. The acceleration results from the universe being first dominated by the slowly expanding region, until it is overtaken by the faster expanding underdense region. The more rapid the transition, the faster the acceleration. In the future, the backreaction models will return to deceleration, asymptotically approaching the empty universe.

The expansion history is illustrated in figure 2 in terms of $H t$. In the $\Lambda$ CDM model, $H t$ grows monotonically from $\frac{2}{3}$ at early times to $H t=0.98$ today, and in the future $H t$ will increase linearly with $t$. At sufficiently early times, backreaction models also have $H t=\frac{2}{3}$. The expansion slows down as the overdense region becomes important, and $H t$ falls below $\frac{2}{3}$. The expansion speeds up when the underdense region takes over; model 1 has $H_{0} t_{0}=0.995$ and model 2 has $H_{0} t_{0}=0.98$. The extra deceleration (compared to the EdS case) before the underdense region takes over makes the change in the expansion rate more drastic, and thus contributes to stronger acceleration. It can also lead to a large value of $\Omega_{\mathrm{m} 0}$ : in model 2 , there is less early deceleration, and correspondingly $\Omega_{\mathrm{m} 0}$ is smaller and the acceleration is weaker than in model 1. This kind of expansion history is quite different from the $\Lambda$ CDM case, but it could be mimicked by a FRW model with suitably exotic matter. We now turn

\footnotetext{
${ }^{1}$ Plots are made with matplotlib [59].
} 


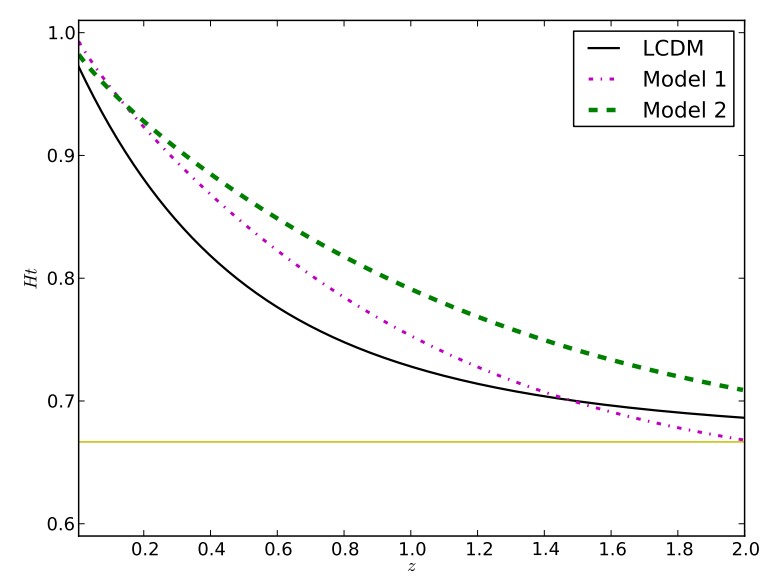

(a)

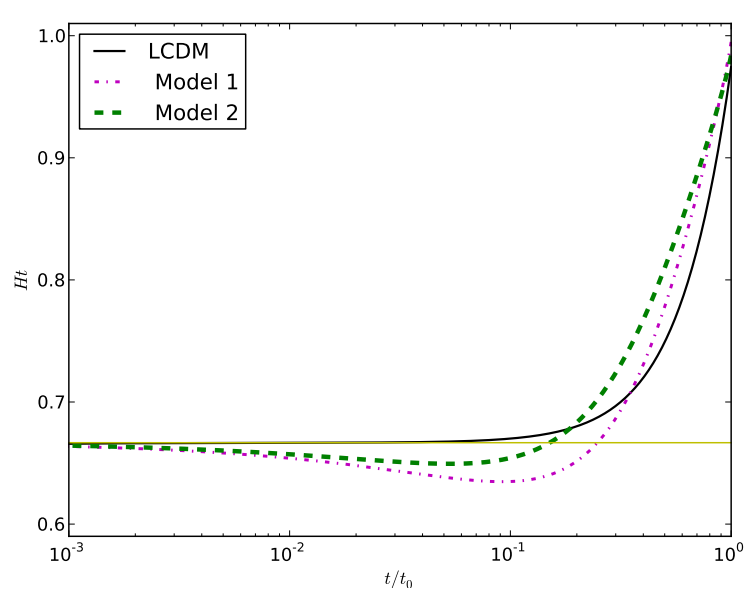

(b)

Figure 2. a) The Hubble parameter times the age of the universe as function of $z$. The horizontal line marks the EdS value $H t=\frac{2}{3}$. b) The same quantity as function of $t / t_{0}$. The value $t / t_{0}=10^{-3}$ corresponds to $z \approx 100$.

to aspects of backreaction which cannot be reproduced by any FRW model.

\section{Signatures of backreaction}

\subsection{Deviation from FRW behaviour}

The density parameters. To understand the evolution of the backreaction models, it is useful to consider the Buchert equations, which describe how the average expansion rate of an irrotational dust universe is related to the variance of the expansion rate, the shear and the spatial curvature [35]:

$$
\begin{aligned}
3 \frac{\ddot{a}}{a} & =-4 \pi G_{\mathrm{N}}\langle\rho\rangle+\mathcal{Q} \\
3 \frac{\dot{a}^{2}}{a^{2}} & =8 \pi G_{\mathrm{N}}\langle\rho\rangle-\frac{1}{2}\left\langle{ }^{(3)} R\right\rangle-\frac{1}{2} \mathcal{Q} \\
0 & =\partial_{t}\langle\rho\rangle+3 \frac{\dot{a}}{a}\langle\rho\rangle,
\end{aligned}
$$

where $\mathcal{Q} \equiv \frac{2}{3}\left(\left\langle\theta^{2}\right\rangle-\langle\theta\rangle^{2}\right)-2\left\langle\sigma^{2}\right\rangle$ is the backreaction variable, \langle\rangle stands for spatial average, $\sigma^{2}$ is the shear scalar and ${ }^{(3)} R$ is the spatial curvature. Dividing (3.1) and (3.2) by $3 H^{2}$, we get [35]

$$
\begin{aligned}
q & \equiv-\frac{1}{H^{2}} \frac{\ddot{a}}{a}=\frac{1}{2} \Omega_{\mathrm{m}}+2 \Omega_{\mathcal{Q}} \\
1 & =\Omega_{\mathrm{m}}+\Omega_{R}+\Omega_{\mathcal{Q}},
\end{aligned}
$$

where $\Omega_{\mathrm{m}} \equiv 8 \pi G_{\mathrm{N}}\langle\rho\rangle /\left(3 H^{2}\right), \Omega_{R} \equiv-\left\langle{ }^{(3)} R\right\rangle /\left(6 H^{2}\right)$ and $\Omega_{\mathcal{Q}} \equiv-\mathcal{Q} /\left(6 H^{2}\right)$ are the density parameters of matter, spatial curvature and the backreaction variable, respectively. In addition to the deceleration parameter $q$, we will use the jerk parameter $j \equiv \frac{1}{H^{3}} \frac{1}{a} \frac{\mathrm{d}^{3} a}{\mathrm{~d} t^{3}}$.

The density parameters as a function of redshift are shown in figure 3. Extra deceleration in model 1 is related to the fact that $\Omega_{\mathrm{m}}>1$ for $z>1$.1. Spatial curvature is correspondingly 


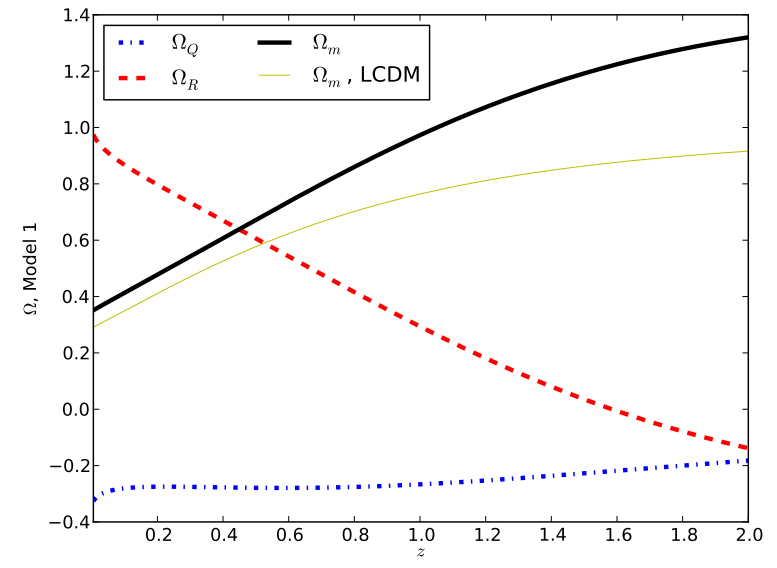

(a)

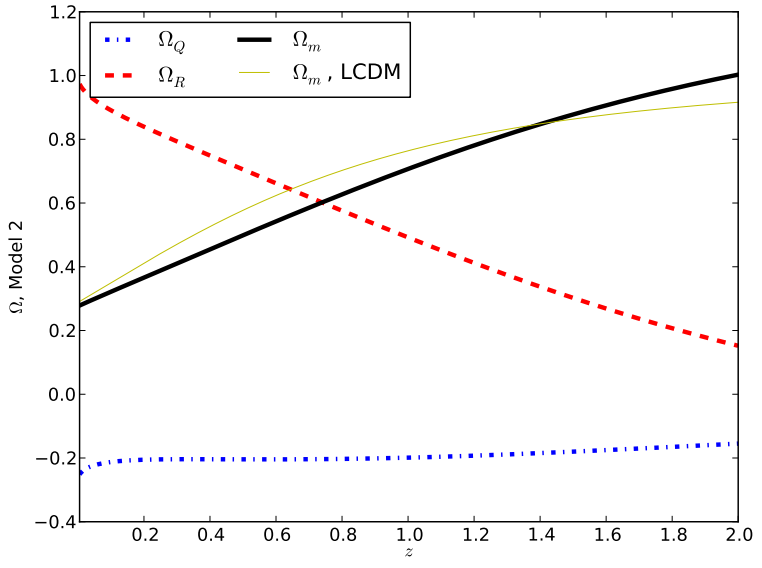

(b)

Figure 3. a) The density parameters (3.5) for a) model 1 and b) model 2 , with $\Omega_{\mathrm{m}}$ for the $\Lambda$ CDM model also plotted.

positive for $z>1.6$, but switches sign as the universe becomes dominated by the underdense region, and the curvature is large today, $\Omega_{R 0}=1.00$. The backreaction variable is also large, $\Omega_{\mathcal{Q} 0}=-0.35$, as required for acceleration. In model 2 , the transition is smoother. The spatial curvature is always negative, and $\Omega_{\mathrm{m}}$ crosses unity earlier, at $z=2.0$.

FRW consistency condition. The backreaction relation (2.8) between distance and expansion rate is different than the FRW relation. The difference can be quantified by a "fitting model" expansion rate $h_{\text {fit }}(z)$, defined as the expansion rate of the spatially flat FRW model with the same distance-redshift relation as the backreaction model. In any FRW model where the expansion rate never passes through zero ${ }^{2}$, distance and expansion rate are related as

$$
d(z)=\frac{1}{\sqrt{\Omega_{K 0}}} \sinh \left(\sqrt{\Omega_{K 0}} \int_{0}^{z} \frac{\mathrm{d} \tilde{z}}{h(\tilde{z})}\right)
$$

where $\Omega_{K 0}$ is the present value of the spatial curvature density parameter $\Omega_{R}$. (In the FRW case, it is conventional to denote the spatial curvature density parameter by $\Omega_{K}$ instead of $\Omega_{R}$.) The spatial curvature of a FRW universe is ${ }^{(3)} R=6 K / a^{2}$, where $K$ is a constant, so $\Omega_{K} \equiv \Omega_{R}=-K /(a H)^{2}$. In the spatially flat case, we have

$$
d(z)=\int_{0}^{z} \frac{\mathrm{d} \tilde{z}}{h(\tilde{z})} .
$$

The fitting model expansion rate is thus

$$
h_{\mathrm{fit}}(z) \equiv \frac{1}{d^{\prime}(z)},
$$

where prime denotes derivative with respect to $z$. The fitting model matter density parameter is correspondingly $\Omega_{\mathrm{mfit}}(z) \equiv \Omega_{\mathrm{m} 0}(1+z)^{3} / h_{\mathrm{fit}}(z)^{2}$.

\footnotetext{
${ }^{2}$ If the expansion rate is not a strictly monotonic function of time, the redshift is not a valid time coordinate everywhere. We assume $h>0$, though $h$ could be allowed to be zero at isolated points.
} 


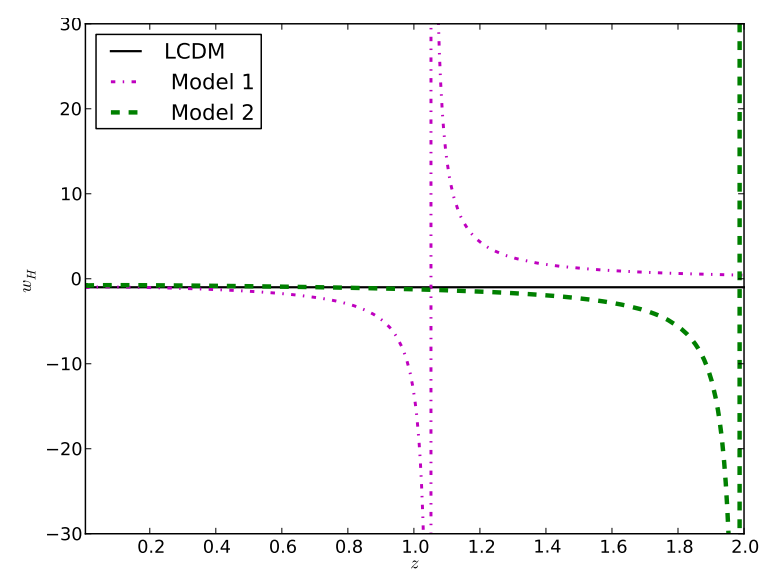

(a)

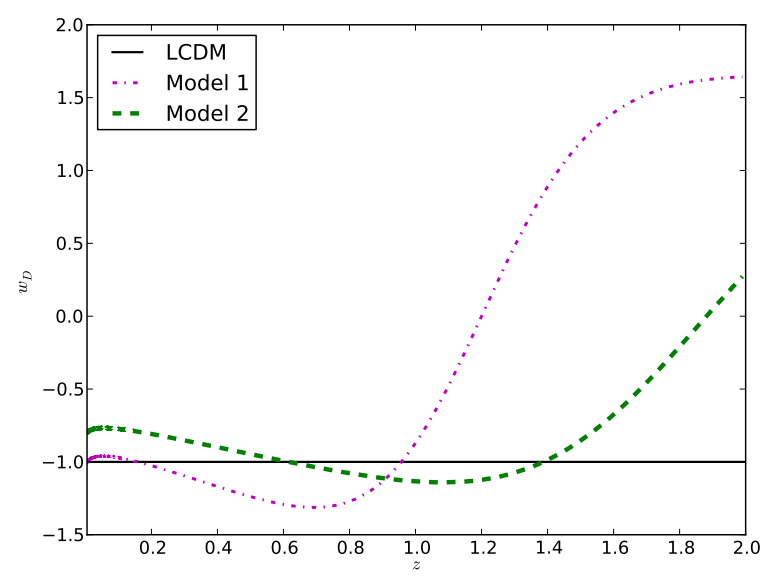

(b)

Figure 4. a) The effective equation of state $w_{H}$ that corresponds to the expansion rate. b) The effective equation of state $w_{D}$ that corresponds to the distance.

The relation (3.6) can be rewritten as [48]

$$
k=\frac{1-h^{2} d^{2}}{d^{2}},
$$

where we have denoted $k \equiv-\Omega_{K 0}=K / H_{0}^{2}$. In the FRW case, $k$ is constant and related to the spatial curvature. However, (3.9) can be taken as the definition of $k$ for any set of observations $h(z)$ and $d(z)$. (We neglect angular variation; in the statistically homogeneous and isotropic case, this is expected to be small for distances larger than the homogeneity scale $[41,42]$.) If the expansion rate and light propagation in the universe are not on average described by a four-dimensional FRW model, $k$ in general varies with redshift [60, 61], and it is not necessarily straightforwardly related to the spatial curvature. The constancy of $k$ was suggested in [48] as a consistency condition for the FRW metric, and the following quantity was introduced to quantify the deviation of $k$ from constant:

$$
\mathscr{C}(z) \equiv-\frac{d^{3}}{2 d^{\prime}} k^{\prime}=1+h^{2}\left(d d^{\prime \prime}-d^{\prime 2}\right)+h h^{\prime} d d^{\prime} .
$$

A non-zero value of $\mathscr{C}$ at any $z$ indicates that the universe cannot be described by a fourdimensional FRW metric.

Using (2.9), we can define a quantity analogous to $k$ for backreaction:

$$
m(z) \equiv-\frac{2 h}{3(1+z) d}\left[h d^{\prime \prime}+h^{\prime} d^{\prime}-(1+z)^{-1} h^{\prime} d\right] .
$$

The quantity $m$ is constant and equal to $\Omega_{\mathrm{m} 0}$ in backreaction, as well as in FRW models where the energy density consists of dust and vacuum energy. In all other FRW models, $m$ depends on $z$. In correspondence with $\mathscr{C}$, we can also define $\mathscr{B} \equiv m^{\prime}$, so that $\mathscr{B}$ vanishes for backreaction. Just as constancy of $k$ (vanishing of $\mathscr{C}$ ) is a test of the FRW metric, constancy of $m$ (vanishing of $\mathscr{B}$ ) provides a test of backreaction. (With the caveat that the relations (2.7) and (2.8) for redshift and distance should be placed on a more rigorous footing.) At small $z, \mathscr{C}$ and $\mathscr{B}$ are less prone to observational errors than $k$ and $m$ in the sense that the 
latter involve the ratio of two quantities that both approach zero as $z$ goes to zero. However, determining derivatives of $d$ is challenging, so it may be easier to use $k$ and $m$, which involve one less derivative than $\mathscr{C}$ and $\mathscr{B}$, respectively.

Effective equations of state. A backreaction model can be equivalently parametrised with two different effective equations of state, one of which corresponds to a FRW model with the same expansion history $h(z)$ and the other to a FRW model with the same distanceredshift relation $d(z)$. Specifically, consider a spatially flat FRW model where the energy density consists of dust (with the same value of $\Omega_{\mathrm{m} 0}$ as in the backreaction model) plus an exotic component. We define the effective expansion equation of state $w_{H}(z)$ as the equation of state of the exotic component, assuming that the FRW model has the same expansion history $h(z)$ as the backreaction model. Correspondingly, the effective distance equation of state $w_{D}(z)$ is defined as the equation of state for which the FRW model has the same distance-redshift relation $d(z)$ as the backreaction model. The effective expansion equation of state is

$$
w_{H}(z) \equiv \frac{2(1+z) \frac{h^{\prime}}{h}-3}{3-3 \Omega_{\mathrm{m}}}=\frac{2 q-1}{3-3 \Omega_{\mathrm{m}}}=\frac{\Omega_{\mathrm{m}}+4 \Omega_{\mathcal{Q}}-1}{3-3 \Omega_{\mathrm{m}}},
$$

where we have in the last equality used (3.4). In $w_{D}(z)$, the quantities $h(z)$ and $\Omega_{\mathrm{m}}(z)$ are replaced by $h_{\text {fit }}(z)$ and $\Omega_{\text {mfit }}(z)$, respectively. (The second and third equality in (3.12) only hold for $w_{H}$.) The functions $w_{H}$ and $w_{D}$ correspond to the equation of state of a new exotic component that would be inferred from observations of the expansion rate and distance, respectively, if they are analysed in the framework of a spatially flat FRW model. This parametrisation is problematic if the effective component at different times decreases and increases the expansion rate $h$ or $h_{\text {fit }}$, because in between the effective energy density passes through zero and the equation of state diverges. It is therefore more informative to use equations of state that correspond to the total effective energy density and pressure, instead of just the exotic component. The effective total expansion equation of state is

$$
w_{H \text { tot }}(z) \equiv \frac{2}{3}(1+z) \frac{h^{\prime}}{h}-1=\frac{2 q-1}{3}=\frac{\Omega_{\mathrm{m}}+4 \Omega_{\mathcal{Q}}-1}{3},
$$

and $w_{D \text { tot }}$ is correspondingly defined with $h$ replaced by $h_{\text {fit }}$. (Again, the second and third equality only hold for $w_{H \text { tot }}$.)

In figure 4 we show the effective equations of state $w_{H}$ and $w_{D}$. The expansion equation of state $w_{H}$ diverges at the redshift where $\Omega_{\mathrm{m}}=1$, which is $z=1.1$ for model 1 and $z=2.0$ for model 2. The distance equation of state $w_{D}$ is finite, it oscillates around -1 at low $z$ and becomes positive at high $z$. This smooth behaviour of $w_{D}$ is not generic. There are well-fitting models for which $\Omega_{\mathrm{mfit}}=1$ in the observable range of redshifts, so $w_{D}$ diverges, demonstrating that an equation of state can be far from -1 and far from constant and yet fit the data well, underlining model-dependence of limits on the equation of state and its change with redshift [11].

The total equations of state $w_{H \text { tot }}$ and $w_{D \text { tot }}$ shown in figure 5 give a clearer picture of how the difference in the distance-expansion rate relation affects the effective equation of state. For both $w_{H \text { tot }}$ and $w_{D \text { tot }}$, there are significant differences between the backreaction models and the $\Lambda \mathrm{CDM}$ model, but $w_{D \text { tot }}$ is closer to the $\Lambda \mathrm{CDM}$ line, in particular for model 2. In the backreaction models, $w_{H \text { tot }}$ crosses the value $-\frac{1}{3}$ at an earlier time (a higher redshift) than in the $\Lambda \mathrm{CDM}$ model, so acceleration starts earlier and is stronger than in the $\Lambda \mathrm{CDM}$ 


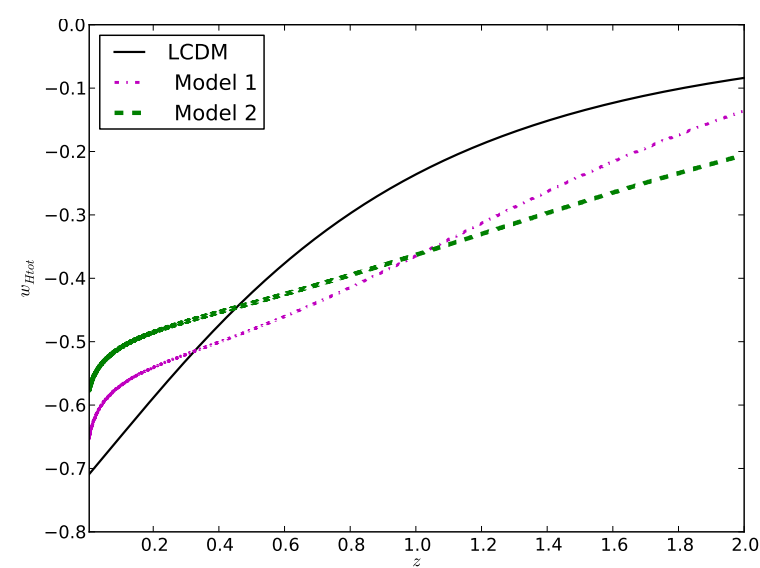

(a)

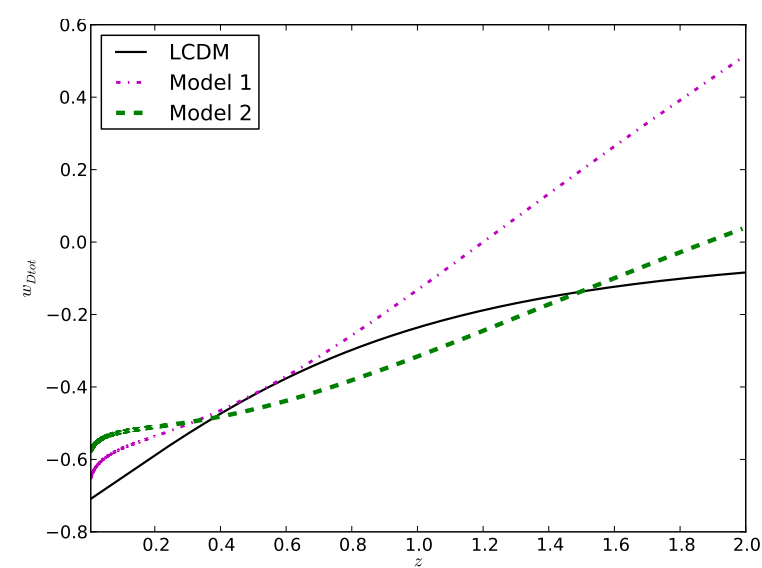

(b)

Figure 5. a) The effective total expansion rate equation of state $w_{H \text { tot }}$. b) The effective total distance equation of state $w_{D \text { tot }}$.

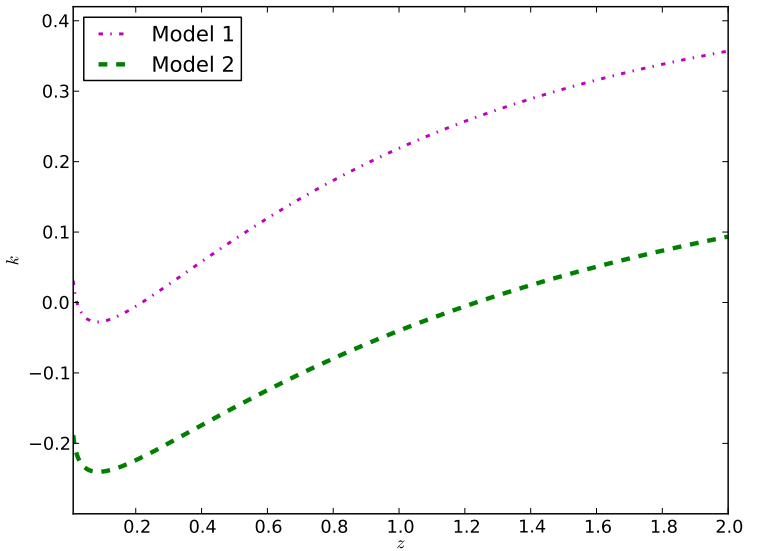

(a)

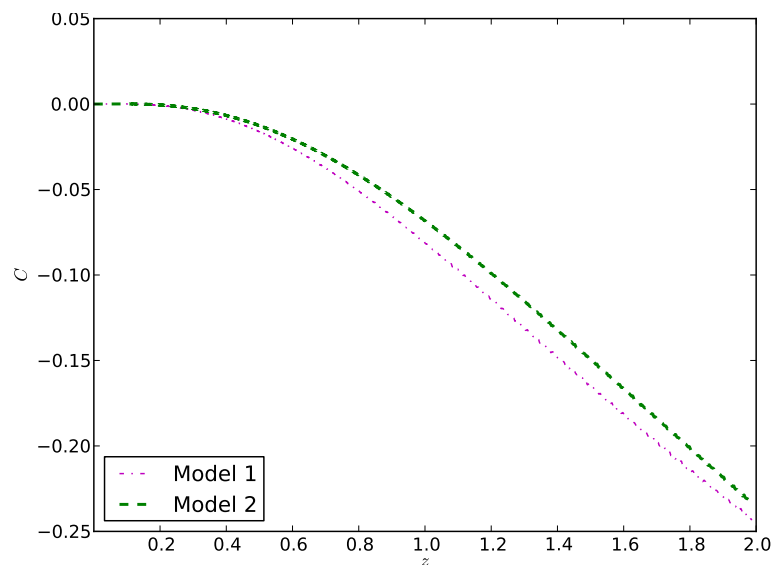

(b)

Figure 6. a) The FRW consistency parameter $k$. b) The FRW consistency parameter $\mathscr{C}$.

model; the trend reverses at small redshifts. This difference is smaller in $w_{D \text { tot }}$. The distance depends on the equation of state via a double integral, so the biasing can be more clearly seen in figure 1a, where crosses and stars mark the magnitude-redshift relation that would correspond to $w_{H \text { tot }}$ in the FRW case. In model 2 , the distance is shifted towards the $\Lambda$ CDM result by the different distance expansion rate relation, but this does not appear to be the case for model 1. (The shifts correspond to changes of $\Delta \chi^{2}=+1$ for model 1 and $\Delta \chi^{2}=-2$ for model 2.) However, to properly evaluate the bias, we should compare backreaction models and $\Lambda \mathrm{CDM}$ models with the same value of $\Omega_{\mathrm{m} 0}$, and for model 1 the value of $\Omega_{\mathrm{m} 0}$ is rather different from its value in $\Lambda \mathrm{CDM}$ model shown.

Violation of the FRW consistency condition. The difference between the equations of state $w_{H \text { tot }}$ and $w_{D \text { tot }}$ is related to the redshift dependence of $k$, shown in figure $6 \mathrm{a}$. For model $1, k$ is positive everywhere except for a small redshift interval close to $z=0.2$, whereas for model $2, k$ is positive at large $z$ and negative for $z<1.3$. At $z=0, k=0.04$ for model 1 and 
$k=-0.20$ for model 2. At $z \gg 1, k$ approaches a constant as backreaction becomes negligible, with asymptotic value -0.2 in model 1 and 0.2 in model 2 , and $|k|<0.5$ at all $z$. A negative $k$ corresponds to $d^{\prime} \geq 1 / h$, so the distance grows faster with increasing $z$ than in the spatially flat FRW model with the same expansion history. In well-fitting toy models, $k$ can have either sign, with a typical magnitude of $0.1 \lesssim|k| \lesssim 1$ at all $z$. We expect the magnitude to be similar in the real universe if the backreaction conjecture is correct. However, a definitive test would require a reliable calculation of the average expansion rate and more careful treatment of light propagation leading to equations (2.7) and (2.8) for redshift and angular diameter distance. In figure $6 \mathrm{~b}$ we show $\mathscr{C}$ that quantifies the redshift dependence of $k$.

In the FRW case, $k=-\Omega_{K 0}$ is given by the spatial curvature, but that is not the case if backreaction is significant. In figure 7 we show ${ }^{(3)} R a^{2} /\left(6 H_{0}^{2}\right)=-a^{2} h^{2} \Omega_{R}$, which in the FRW case would be equal to $k$. For the backreaction models its evolution is quite different from that of $k$, demonstrating that in general $k$ does not describe spatial curvature. Even at large $z$ where backreaction is small and both ${ }^{(3)} R a^{2} /\left(6 H_{0}^{2}\right)$ and $k$ are constant, their values (or even signs) do

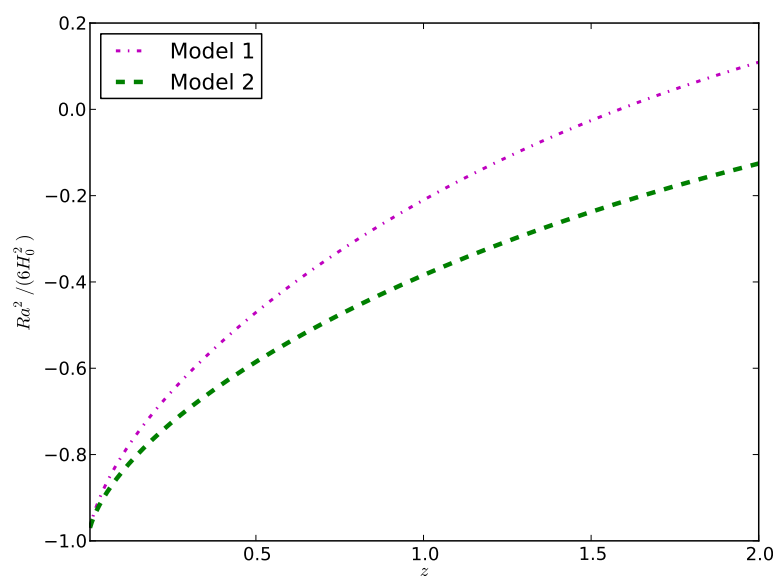

Figure 7. The quantity ${ }^{(3)} R a^{2} /\left(6 H_{0}^{2}\right)$ that would be constant and equal to $k$ in a FRW universe. not in general agree.

\subsection{Low redshift expansion}

Distance bias. Let us consider the change of the distance-expansion rate relation analytically. Unlike in the FRW case, there is no closed form solution for the distance in terms of the expansion rate, so we consider a series expansion around $z=0$. Given an expansion history $h(z)$, the FRW distance-expansion rate relation (3.6) gives

$$
d_{A} \simeq z-\frac{1}{2}\left(3+q_{0}\right) z^{2}+\frac{1}{6}\left(11+7 q_{0}+3 q_{0}^{2}-j_{0}+\Omega_{K 0}\right) z^{3}+\mathcal{O}\left(z^{4}\right),
$$

where $q_{0}=h_{0}^{\prime}-1$ and $j_{0}=h_{0}^{\prime \prime}+\left(h_{0}^{\prime}-1\right)^{2}$. Solving for the distance from the backreaction relation (2.9) instead, we obtain

$$
d_{A} \simeq z-\frac{1}{2}\left(3+q_{0}\right) z^{2}+\frac{1}{6}\left(12+8 q_{0}+3 q_{0}^{2}-j_{0}-\frac{3}{2} \Omega_{\mathrm{m} 0}\right) z^{3}+\mathcal{O}\left(z^{4}\right) .
$$

Biasing of the distance shows up only at order $z^{3}$, like spatial curvature. This can be easily seen from (2.9), as the only difference between FRW and backreaction is the pressure term on the right-hand side of the equation, and it doesn't contribute until at order $z^{3}$. The difference at order $z^{3}$ corresponds to the jerk parameter being shifted as $j_{0} \rightarrow j_{0}-\left(1+q_{0}\right)+$ $\frac{3}{2} \Omega_{\mathrm{m} 0}+\Omega_{K 0}$. If $\Omega_{K 0}=0$, we can write this as $j_{0} \rightarrow j_{0}-\frac{3}{2}\left(1-\Omega_{\mathrm{m} 0}\right)\left(1+w_{H 0}\right)$. So, assuming that the effective energy density of the extra component is positive $\left(\Omega_{\mathrm{m} 0}<1\right)$, the distance for small $z$ is longer in the backreaction case than in the FRW case if $w_{H}>-1$ and shorter if $w_{H}<-1$. In other words, the distance is biased towards the $\Lambda$ CDM model [38]. If the 
expansion history is exactly that of the $\Lambda$ CDM model (plus possibly spatial curvature), the distance is the same as in the FRW case.

It has been noted that because of the bias, the observed distances do not necessarily imply that the expansion would have accelerated, unlike in the FRW case [1, 38, 41, 42]. The expansion (3.15) shows that if low redshift observations are analysed with a power series expansion up to second order in $z$ and the coefficient of the second order term of $d_{A}$ is found to be larger than $-\frac{3}{2}$, we can conclude that the volume expansion rate has accelerated, just as in the FRW case. (For observations extending to high redshifts, the biasing cannot be estimated so simply, as we have seen.) The small redshift expansion is limited by the fact that the mean quantities $z$ and $d_{A}$ are not expected to describe the physical redshift and distance over distances smaller than the homogeneity scale, and an expansion of local quantities in terms of the redshift is not useful due to large local variations. This issue is present even if the average properties of the universe are on large scales well described by the FRW model, and deviations from the mean expansion on short distances are usually known under the name peculiar velocities; see [62] on limitations of the series expansion. The homogeneity scale of $100 \mathrm{Mpc}$ corresponds to $z=0.02$, and data on such small redshifts is usually dropped due to large local variations. However, the homogeneity scale refers to a three-dimensional average, and one-dimensional integrals like the redshift and the distance may be more sensitive to inhomogeneity, depending on the filamentarity of the matter distribution.

FRW consistency parameter $k$. The series expansion also sheds some light on the magnitude of the FRW consistency parameter $k$. Using the expansion (3.15), the FRW consistency parameter (3.9) at $z=0$ can be written as

$$
k_{0}=-q_{0}-1+\frac{3}{2} \Omega_{\mathrm{m} 0}=\Omega_{\mathrm{m} 0}-2 \Omega_{\mathcal{Q} 0}-1 .
$$

The amplitude of $k_{0}$ equals the deviation from the $\Lambda$ CDM relation between $q_{0}$ and $\frac{3}{2} \Omega_{\mathrm{m} 0}$. With (3.8) and (3.9), we can write $k=\left(1-h^{2} / h_{\text {fit }}^{2}\right) d^{-2}$, so for $z \ll 1$ we have $h / h_{\text {fit }} \simeq$ $1-\frac{1}{2} k_{0} z^{2}$. Observationally, we know model-independently that, roughly, $0.2 \lesssim \Omega_{\mathrm{m} 0} \lesssim$ $0.4[43,44,63-66]$. Values of $q_{0}$ quoted in the literature depend strongly on the assumed parametrisation $[8,9,11,13]$, and are heavily based on distance measurements, whereas it is the expansion rate which is of interest here. From measurements of the expansion rate [15$21,23-26]$ it is not yet possible to determine $q_{0}$ reliably, but $\left|q_{0}\right| \lesssim 1$ is a reasonable estimate. Without a reliable calculation of backreaction, it is not possible to definitely exclude the possibility that $1+q_{0}$ and $-\frac{3}{2} \Omega_{\mathrm{m} 0}$ accurately cancel. However, as there does not appear to be any reason for such a close cancellation, $k_{0}$ can be expected to be of the same order as $1+q_{0}$ and $\Omega_{\mathrm{m} 0}$ or slightly smaller, roughly $0.1 \lesssim\left|k_{0}\right| \lesssim 1$. In the toy model, this is the typical magnitude at all $z$, though in model $1, k_{0}$ is an order of magnitude smaller than $1+q_{0}$ and $\Omega_{\mathrm{m} 0}$.

For FRW models, the value $k=-\Omega_{K 0}$ is tightly constrained to be $|k| \lesssim 0.01$ [67]. The strong constraint on $k$ is related to the fact that $k$ has two roles in FRW models. It changes the expansion rate by $h^{2} \rightarrow h^{2}-k(1+z)^{2}$ and affects the distance by changing the spatial geometry, which is manifested in the sinh behaviour in (3.6). (From the point of view of the light propagation equation (2.9), the latter effect follows from the former.) In [68] these effects were studied separately by introducing two parameters, one that only modifies the spatial geometry and another that only alters the expansion rate. Taking the energy density to consist of dust and vacuum energy, limits for the geometrical constant are $-0.4<k_{0}<1.2$ 
from the Union2 data alone and $-0.1<k_{0}<0.2$ when other data are included. (We quote error bars as $68 \%$ C.L. limits and ranges as $95 \%$ C.L. limits.) Using the SDSS SN dataset instead of Union2, the limits change somewhat, to $-0.8<k_{0}<1.2$ for SN data alone and $-0.3<k_{0}<0.0$ with other datasets added. The limits for the constant which modifies the expansion rate are similar when only the SN data are used, and a factor of 6 tighter with other data added. If the two curvature parameters are taken to be the same, the limit reduces to $\left|k_{0}\right| \lesssim 0.01$ for the full dataset (again assuming dust and vacuum energy), regardless of which set of SNe is considered. With current CMB data, a similar constraint can be achieved without any other datasets due to the sensitivity of the late Integrated Sachs-Wolfe (ISW) effect and lensing to the expansion rate at late times [69].

In general, backreaction does not reduce to a modification of the FRW spatial curvature constant, and if it does, it follows from (2.9) that the parameter appearing in the sinh term and the expansion rate is the same, unlike argued in [68]. If the average spatial curvature (or rather $\left\langle{ }^{(3)} R\right\rangle+3 \mathcal{Q}$; see eq. 40 of [41]) is proportional to $a^{-2}=(1+z)^{2}$, the relation between distance and expansion rate solved from (2.9) contains a sinh term. If the relative deviation from the EdS behaviour drops down faster at large redshift, the modification to the distance will be less extreme. The tight constraint on spatial curvature in FRW models (strongly driven by the measurement of the angular diameter distance to last scattering from the CMB) shows that the expansion rate has to approach the EdS case faster than $(1+z)^{2} /\langle\rho\rangle \propto(1+z)^{-1}$ as $z$ grows. This constrains the value of $k(z)$ at high $z$, not at $z=0$.

To obtain a model-independent measure of $k$, distance and expansion rate have to be observed independently. The clustering of large-scale structure, in particular the radial part of the BAO, contains detailed information about the expansion rate [15-21], and the expansion rate can be also determined from galaxy ages [23-26]. The resulting constraint is roughly $|k| \lesssim 1[70,71]$, much weaker than when the FRW model is assumed, though the analyses have not included all current data. The constraint is within an order of magnitude of the expected amplitude of $k$ from backreaction, so near future observations may provide useful constraints.

There have been tests of the distance duality relation $D_{L}=(1+z)^{2} D_{A}$, where the angular diameter distance data is obtained from BAO or galaxy ages [72]. The FRW distanceexpansion rate relation (3.6) has been used to convert the expansion rate from galaxy ages and the radial $\mathrm{BAO}$ component into distance. Apparent violation of the duality relation could therefore be indicative of the failure of the FRW distance-expansion rate relation instead. No statistically significant discrepancy has been found.

\subsection{Other observations}

Expansion rate. We have fitted the toy model only to the SN distance data. Let us now consider other observables. In figure 8 we show the expansion rate $h(z)=H(z) / H_{0}$ for the two backreaction models and the $\Lambda \mathrm{CDM}$ model, along with data points from large-scale structure [15-20] and galaxy ages [24-26]. We have adopted the value $H_{0}=72 \mathrm{~km} / \mathrm{s} / \mathrm{Mpc}$, and the points could be moved up or down together by the uncertainty in $H_{0} \cdot{ }^{3}$ The assigned errors of some galaxy age measurements have been criticised as too small [71], there is some

\footnotetext{
${ }^{3}$ The value $H_{0}=73.8 \pm 2.4 \mathrm{~km} / \mathrm{s} / \mathrm{Mpc}$ is quoted in [65], and the more recent study [66] gives $H_{0}=74.3 \pm 2.1$. We allow for the possibility of unaccounted systematics and simply take the upper and lower ranges obtained with the three different calibrators used in [65], $64 \mathrm{~km} / \mathrm{s} / \mathrm{Mpc}<H_{0}<81 \mathrm{~km} / \mathrm{s} / \mathrm{Mpc}$, or $H_{0}=72 \pm 4$ $\mathrm{km} / \mathrm{s} / \mathrm{Mpc}$. The precise central value and error bars have little effect on our results.
} 
model-dependence in the large-scale structure analysis and the large-scale structure data points are not all independent. Therefore, the data points and their errors in figure 8 are only indicative. The goodness-offit is $\chi^{2}=17$ for the $\Lambda$ CDM model, $\chi^{2}=18$ for model 1 and $\chi^{2}=19$ for model 2 , for 37 data points (for either choice of stellar population synthesis model used in [25]). This agrees with the visual impression that differences between the $\Lambda$ CDM model and the backreaction models are not large compared to the error bars.

Age of the universe. We can also consider the age of the universe relative to the Hubble parameter. Globular clusters give the $95 \%$ lower limit $t_{0}>11.2 \mathrm{Gyr}$ and the best-fit age 13.4 Gyr [73]. Taking $t_{0}=$

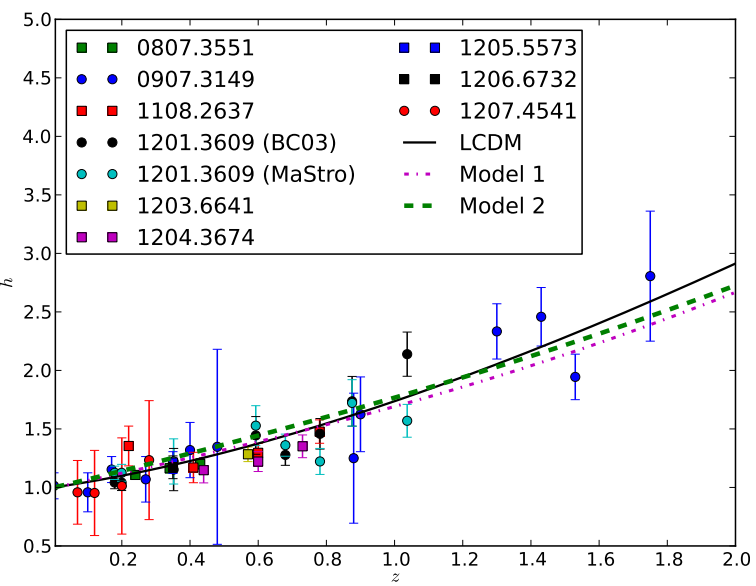

Figure 8. The Hubble parameter relative to its present value. The data points are from large-scale structure (boxes) [15-20] and galaxy ages (circles) [24-26]. Numbers refer to arXiv identifiers, labels BC03 and MaStro refer to stellar population synthesis models used in [25]. $13.4 \pm 1.1 \mathrm{Gyr}$ and $H_{0}=72 \pm 4 \mathrm{~km} / \mathrm{s} / \mathrm{Mpc}$ would give $H_{0} t_{0}=0.99 \pm 0.14$. However, the distribution of $t_{0}$ is not Gaussian, and the "firm upper limit" is given as $t_{0}<21$ Gyr. Considering only the lower limit $t_{0} \geq 11.2$ Gyr and fixing $H_{0}=72 \mathrm{~km} / \mathrm{s} / \mathrm{Mpc}$ gives $H_{0} t_{0} \geq 0.82$. There is a more recent and higher age estimate of $14.61 \pm 0.8 \mathrm{Gyr}$, which is however based only on a single star [74]. With $H_{0}=72$ $\mathrm{km} / \mathrm{s} / \mathrm{Mpc}$, this would give $H_{0} t_{0}=1.08 \pm 0.06$. Figure 2 shows that the backreaction models satisfy the constraint $H_{0} t_{0} \geq 0.82$ easily: $H t$ is close to unity today because the volume is almost completely taken up by underdense region, which is almost completely empty. In the real universe, the value of $H_{0} t_{0}$ is not expected to be as close to unity as in the toy model. In backreaction models, $H t<1$ is a general prediction, provided matter can be treated as dust and vorticity is not important $[42,75]$. Were the value $H_{0} t_{0}=1.08$ to be reliably confirmed with more precision, it would rule out the backreaction conjecture. (In combination with other cosmological datasets, it would also rule out the $\Lambda$ CDM model.)

Distance to the last scattering surface. The main sensitivity of the CMB to late-time evolution of the universe is via the angular diameter distance, which affects the position of the acoustic peaks in multipole space [43]. The Union2.1 data gives distances relative to $H_{0}$, whereas the $\mathrm{CMB}$ peak position gives the distance to the redshift of the last scattering surface at $z_{*} \approx 1090$ in physical units ${ }^{4}$. Therefore, any model fitted to only the Union2.1 data is consistent with either any value of $D_{A}\left(z_{*}\right)$ or any value of $H_{0}$, but the combination of all three measurements imposes constraints. Model-independent analysis of WMAP sevenyear data and SPT data gives $D_{A}\left(z_{*}\right)=12.7 \pm 0.2 \mathrm{Mpc}[43,44]$. (The precision of current observations of $D_{A}\left(z_{*}\right)$ relative to the sound horizon from the Planck satellite is a factor of 1.7 better, but they are also more model-dependent [69].) With $H_{0}=72 \pm 4 \mathrm{~km} / \mathrm{s} / \mathrm{Mpc}$, this translates into $d\left(z_{*}\right)=3.3_{-0.2}^{+0.3}$. We have simply combined the $68 \%$ ranges, so the error is rather conservative. For the $\Lambda$ CDM best-fit model we have $d\left(z_{*}\right)=3.3$, whereas models 1 and 2 give $d\left(z_{*}\right)=0.72$ and $d\left(z_{*}\right)=1.6$, respectively, in strong disagreement with observation.

\footnotetext{
${ }^{4}$ More precisely, the peak position fixes $D_{A}\left(z_{*}\right)$ relative to the sound horizon at last scattering, but the sound horizon is also determined by the CMB data.
} 
The fact that the $\Lambda \mathrm{CDM}$ model fitted to distance data at small redshift correctly predicts the distance to $z \approx 1090$ is indicative of the fact that any model that fits the low $z$ observations and rapidly approaches EdS behaviour at $z \gtrsim 2$ (with a radiation contribution at high $z$ ) will fit all data also at higher $z$. This is not the case for our toy models. The expansion rate at early times is smaller than in the EdS case, so there is less overall expansion, leading to shorter distances. This is related to the fact that as the toy model is constructed as the union of two FRW models, the contribution of spatial curvature relative to matter density decreases slowly with increasing redshift. Figure $2 \mathrm{~b}$ shows that the expansion rate in the toy model deviates noticeably from the EdS behaviour already at $10^{-2} t_{0}$, corresponding to less than a billion years of age, a time when the $\Lambda \mathrm{CDM}$ expansion rate is indistinguishable from the EdS case. The problem could be fixed by tweaking the expansion history to make the transition from EdS behaviour sharper, but that would be taking the toy model too seriously. If backreaction is significant in the real universe, the timescale of the change from EdS behaviour is related to structure formation. In a semirealistic model based on statistics of structure formation the timescale is not dissimilar to that shown in figure 2 [47] (see figure 1 in [1]). What happens in the fully realistic case remains to be determined.

In addition to the peak positions, with current CMB data the lensing and the ISW effect (in the backreaction context more properly called the Rees-Sciama effect due to its non-linear origin) have become important cosmological probes, as they are affected by the expansion rate at late times $[69,76]$. Even if backreaction is significant, such secondary anisotropies are expected to be small in absolute terms despite large local variations in the geometry, because of statistical homogeneity and isotropy [41, 42, 77], but their magnitude relative to the primary anisotropy remains to be determined. The ISW contribution to the overall two-point correlation function does not significantly deviate from the $\Lambda$ CDM prediction, but there is excess power along lines of sight that cross extrema of the density field [2], and there are also other anomalies on large angular scales [3].

\section{Conclusion}

Summary. Backreaction, i.e. change of the average expansion rate due to structure formation, is a possible explanation for the observation that the expansion rate and distance are larger than predicted by homogeneous and isotropic models with ordinary matter and ordinary gravity. So far there is no fully realistic calculation of the effect of structure formation on the average expansion rate of the universe. Nevertheless, it is possible to test backreaction by comparing measurements of distance and expansion rate, as their relation is different than in the FRW case [41, 42].

We have presented a toy model of backreaction where the expansion rate has realistic features. We have shown that the model provides a good fit to the Union2.1 SN data, even though the expansion history is quite different from the $\Lambda \mathrm{CDM}$ model. The model also illustrates the feature that the effective equation of state inferred from distance measurements is different from the effective equation of state that corresponds to the expansion rate. We find that the deviation from the FRW distance-expansion rate relation in the toy model is slightly below current observational constraints. The order of magnitude is expected to be the same in the real universe if backreaction is signicant. This provides a distinct signature that may be used to put meaningful constraints on backreaction with improved observations of the expansion rate in the near future. We have also defined a distance-expansion rate consistency condition for backreaction that may be used to test backreaction. However, 
improved theoretical treatment is needed for definite tests, and the average expansion rate, light propagation and structure formation all need to be understood better.

\section{Acknowledgments}

SR thanks Bruno Leibundgut for correspondence, and we thank Phil Bull for pointing out a binning error in a previous version of figure 1.

\section{References}

[1] T. Buchert and S. Räsänen, Backreaction in late-time cosmology, Ann. Rev. Nucl. Part. Sci. 62 (2012) 57 [arXiv:1112.5335 [astro-ph.CO]]

[2] T. Giannantonio, R. Crittenden, R. Nichol and A. J. Ross, The significance of the integrated Sachs-Wolfe effect revisited, Mon. Not. Roy. Astron. Soc. 426 (2012) 2581 [arXiv:1209.2125 [astro-ph.CO]] S. Flender, S. Hotchkiss and S. Nadathur, The stacked ISW signal of rare superstructures in $\Lambda C D M$, JCAP02(2013)013 [arXiv:1212.0776 [astro-ph.CO]]

C. Hernandez-Monteagudo and R.E. Smith, On the signature of nearby superclusters and voids in the Integrated Sachs-Wolfe effect [arXiv:1212.1174 [astro-ph.CO]]

S. Ilic, M. Langer and M. Douspis, On the detection of the integrated Sachs-Wolfe effect with stacked voids [arXiv:1301.5849 [astro-ph.CO]]

Y.-C. Cai, M.C. Neyrinck, I. Szapudi, S. Cole and C.S. Frenk, A Detection of the Cold Imprint of Voids on the Microwave Background Radiation [arXiv:1301.6136 [astro-ph.CO]]

P.A.R. Ade et al. [Planck Collaboration], Planck 2013 results. XIX. The integrated Sachs-Wolfe effect, [arXiv:1303.5079 [astro-ph.CO]]

[3] C.J. Copi, D. Huterer, D.J. Schwarz and G.D. Starkman, Large angle anomalies in the CMB, Adv. Astron. 2010 (2010) 847541 [arXiv:1004.5602 [astro-ph.CO]]

P.A.R. Ade et al. [Planck Collaboration], Planck 2013 results. XXIII. Isotropy and Statistics of the $C M B$ [arXiv:1303.5083 [astro-ph.CO]]

[4] J. Einasto et al., Superclusters of galaxies from the 2dF redshift survey. I. The catalogue, Astron. $\mathcal{E}$ Astrophys. 462 (2007) 811 [arXiv:astro-ph/0603764]

J. Einasto et al., Superclusters of galaxies from the $2 d F$ redshift survey. II. Comparison with simulations, Astron. \& Astrophys. 462 (2007) 397 [arXiv:astro-ph/0604539]

J. Einasto et al., Luminous superclusters: remnants from inflation, Astron. ES Astrophys. 459 (2006)

L1 [arXiv:astro-ph/0605393]

J. Einasto, Formation of the Supercluster-Void Network [arXiv:astro-ph/0609686]

[5] F. Sylos Labini, Characterizing the large scale inhomogeneity of the galaxy distribution, AIP Conf.

Proc. 1241 (2010) 981-990 [arXiv:0910.3833 [astro-ph.CO]]

F. Sylos Labini and L. Pietronero, The complex universe: recent observations and theoretical challenges, J. Stat. Mech. (2010) P11029 [arXiv:1012.5624 [astro-ph.CO]]

F. Sylos Labini, Inhomogeneities in the universe, Class. Quant. Grav. 28 (2011) 164003 [arXiv:1103.5974 [astro-ph.CO]]

[6] C.Y. Yaryura, C.M. Baugh and R.E. Angulo, Are the 2dFGRS superstructures a problem for hierarchical models?, Mon. Not. Roy. Astron. Soc. 413 (2011) 1311 [arXiv:1003.4259 [astro-ph.CO]] D.N.A. Murphy, V.R. Eke and C.S. Frenk, Connected structure in the 2dFGRS, Mon. Not. Roy. Astron. Soc. 413 (2011) 2288 [arXiv:1010.2202 [astro-ph.CO]]

[7] C. Contreras et al. [WiggleZ Collaboration], The WiggleZ Dark Energy Survey: measuring the cosmic growth rate with the two-point galaxy correlation function, Mon. Not. Roy. Astron. Soc. 430 (2013) 924 [arXiv:1302.5178 [astro-ph.CO]]

[8] U. Alam, V. Sahni and A.A. Starobinsky, Is there Supernova Evidence for Dark Energy Metamorphosis?, Mon. Not. Roy. Astron. Soc. 354 (2004) 275 [arXiv:astro-ph/0311364] U. Alam, V. Sahni and A.A. Starobinsky, The case for dynamical dark energy revisited, JCAP06(2004)008 [arXiv:astro-ph/0403687] 
J. Jönsson, A. Goobar, R. Amanullah and L. Bergström, No evidence for Dark Energy

Metamorphosis?, JCAP09(2004)007 [arXiv:astro-ph/0404468]

U. Alam, V. Sahni and A.A. Starobinsky, Rejoinder to "No Evidence of Dark Energy Metamorphosis", astro-ph/0404468, [arXiv:astro-ph/0406672]

[9] M.V. John, Bayesian model-independent evaluation of expansion rates of the universe, Astrophys. Space Sci. 330 (2010) 7 [arXiv:1003.6115 [astro-ph.CO]]

A.C.C. Guimaraes and J.A.S. Lima, Could the cosmic acceleration be transient? A cosmographic evaluation, Class. Quant. Grav. 28 (2011) 125026 [arXiv:1005.2986 [astro-ph.CO]]

A. Shafieloo, V. Sahni and A.A. Starobinsky, Is cosmic acceleration slowing down?, Phys. Rev. D80 (2009) 101301 [arXiv:0903.5141 [astro-ph.CO]]

$\mathrm{P}$. Wu and H.W. Yu, Is the present cosmic expansion decelerating? [arXiv:1012.3032 [astro-ph.CO]] G.-B. Zhao, R.G. Crittenden, L. Pogosian and X. Zhang, Examining the evidence for dynamical dark energy, Phys. Rev. Lett. 109 (2012) 171301 [arXiv:1207.3804 [astro-ph.CO]]

[10] L. Perivolaropoulos and A. Shafieloo, Bright High z SnIa: A Challenge for LCDM?, Phys. Rev. D79 (2009) 123502 [arXiv:0811.2802 [astro-ph]]

[11] B.A. Bassett, M. Kunz, J. Silk and C. Ungarelli, A late-time transition in the cosmic dark energy?, Mon. Not. Roy. Astron. Soc. 336 (2002) 1217 [arXiv:astro-ph/0203383]

P.S. Corasaniti, M. Kunz, D. Parkinson, E.J. Copeland and B.A. Bassett, The foundations of observing dark energy dynamics with the Wilkinson Microwave Anisotropy Probe, Phys. Rev. D70 (2004) 083006 [arXiv:astro-ph/0406608]

S. Hannestad and E. Mörtsell, Cosmological constraints on the dark energy equation of state and its evolution, JCAP09(2004)001 [arXiv:astro-ph/0407259]

B.A. Bassett, P.S. Corasaniti and M. Kunz, The essence of quintessence and the cost of compression, Astrophys. J. 617 (2004) L1 [arXiv:astro-ph/0407364]

C. Stephan-Otto, Optimized supernova constraints on dark energy evolution, Phys. Rev. D74 (2006) 023507 [arXiv:astro-ph/0605403]

Y. Gong, B. Wang and R.-g. Cai, Probing the cosmic acceleration from combinations of different data sets, JCAP04(2010) 019 [arXiv:1001.0807 [astro-ph.CO]]

[12] G.R. Bengochea, Supernova light-curve fitters and Dark Energy, Phys. Lett. B696 (2011) 5 [arXiv:1010.4014 [astro-ph.CO]]

Z. Li, P. Wu and H. Yu, Probing the course of cosmic expansion with a combination of observational data, JCAP11(2010)031 [arXiv:1011.2036 [gr-qc]]

M.C. March, R. Trotta, P. Berkes, G.D. Starkman and P.M. Vaudrevange, Improved constraints on cosmological parameters from SNIa data, Mon. Not. Roy. Astron. Soc. 418 (2011) 2308 [arXiv:1102.3237 [astro-ph.CO]]

R. Giostri, M. Vargas dos Santos, I. Waga, R.R.R. Reis, M.O. Calvao and B.L. Lago, From cosmic deceleration to acceleration: new constraints from SN Ia and BAO/CMB, JCAP03 (2012) 027 [arXiv:1203.3213 [astro-ph.CO]]

[13] C. Shapiro and M.S. Turner, What Do We Really Know About Cosmic Acceleration?, Astrophys. J. 649 (2006) 563 [arXiv:astro-ph/0512586]

Y. Gong and A. Wang, Observational constraints on the acceleration of the Universe, Phys. Rev. D73 (2006) 083506 [arXiv:astro-ph/0601453]

Ø. Elgarøy and T. Multamäki, Bayesian analysis of Friedmannless cosmologies, JCAP09(2006)002 [arXiv:astro-ph/0603053]

C. Cattoën and M. Visser, Cosmography: Extracting the Hubble series from the supernova data [arXiv:gr-qc/0703122]

M. Seikel and D.J. Schwarz, How strong is the evidence for accelerated expansion?, JCAP02(2008)007 [arXiv:0711.3180 [astro-ph]]

M. Seikel and D.J. Schwarz, Model- and calibration-independent test of cosmic acceleration, JCAP02(2009)024 [arXiv:0810.4484 [astro-ph]]

E. Mörtsell and C. Clarkson, Model independent constraints on the cosmological expansion rate, JCAP01(2009)044 [arXiv:0811.0981 [astro-ph]]

A.C.C. Guimarães, J.V. Cunha and J.A.S. Lima, Bayesian Analysis and Constraints on Kinematic Models from Union SNIa, JCAP10(2009)010 [arXiv:0904.3550 [astro-ph.CO]]

M. Visser and C. Cattoën, Cosmographic analysis of dark energy, 2010, Proc. 7th International 
Heidelberg Conference on Dark Matter in Astro and Particle Physics, eds. H.V. Klapdor-Kleingrothaus and I.V. Krivosheina [arXiv:0906.5407 [gr-qc]]

P. Serra, A. Cooray, D.E. Holz, A. Melchiorri, S. Pandolfi and D. Sarkar, No Evidence for Dark Energy Dynamics from a Global Analysis of Cosmological Data, Phys. Rev. D80 (2009) 121302

[arXiv:0908.3186 [astro-ph.CO]]

R.-G. Cai, Q. Su and H.-B. Zhang, Probing the dynamical behavior of dark energy, JCAP04(2010) 012 [arXiv:1001.2207 [astro-ph.CO]]

S. Wang, X.-D. Li and M. Li, Exploring the Latest Union2 SNIa Dataset by Using Model-Independent Parametrization Methods, Phys. Rev. D83 (2011) 023010 [arXiv:1009.5837 [astro-ph.CO]]

J. Park, C.-G. Park and J.-c. Hwang, Analysis of recent type Ia supernova data based on evolving dark energy models, Phys. Rev. D84 (2011) 023506 [arXiv:1011.1723 [astro-ph.CO]]

A.V. Pan and U. Alam, Reconstructing Dark Energy : A Comparison of Cosmological Parameters, [arXiv:1012.1591 [astro-ph.CO]]

R.-G. Cai and Z.-L. Tuo, Detecting the cosmic acceleration with current data, Phys. Lett. B706 (2011) 116 [arXiv:1105.1603 [astro-ph.CO]]

X.-D. Li, S. Li, S. Wang, W.-S. Zhang, Q.-G. Huang and M. Li, Probing Cosmic Acceleration by Using the SNLS3 SNIa Dataset, JCAP07(2011) 011 [arXiv:1106.4116 [astro-ph.CO]]

A. Shafieloo, A.G. Kim and E.V. Linder, Gaussian Process Cosmography, Phys. Rev. D85 (2012) 123530 [arXiv:1204.2272 [astro-ph.CO]]

A. Aviles, C. Gruber, O. Luongo and H. Quevedo, Cosmography and constraints on the equation of state of the Universe in various parametrizations, [arXiv:1204.2007 [astro-ph.CO]] Phys. Rev. D86 (2012) 123516

[14] B.A. Bassett and R. Hlozek, Baryon Acoustic Oscillations, Dark Energy: Observational and Theoretical Approaches, ed. P. Ruiz-Lapuente (Cambridge University Press, 2010), p. 246 [arXiv:0910.5224 [astro-ph.CO]]

[15] E. Gaztañaga, A. Cabré A and L. Hui, Clustering of Luminous Red Galaxies IV: Baryon Acoustic Peak in the Line-of-Sight Direction and a Direct Measurement of $H(z)$ [arXiv:0807.3551 [astro-ph]]

[16] C. Blake et al., The WiggleZ Dark Energy Survey: measuring the cosmic expansion history using the Alcock-Paczynski test and distant supernovae, Mon. Not. Roy. Astron. Soc. 418 (2011) 1725 [arXiv:1108.2637 [astro-ph.CO]]

[17] B.A. Reid et al., The clustering of galaxies in the SDSS-III Baryon Oscillation Spectroscopic Survey: measurements of the growth of structure and expansion rate at $z=0.57$ from anisotropic clustering, Mon. Not. Roy. Astron. Soc. 426 (2012) 2719 [arXiv:1203.6641 [astro-ph.CO]]

[18] C. Blake et al., The WiggleZ Dark Energy Survey: Joint measurements of the expansion and growth history at $z<1$, Mon. Not. Roy. Astron. Soc. 425 (2012) 405 [arXiv:1204.3674 [astro-ph.CO]]

[19] C.-H. Chuang and Y. Wang, Using Multipoles of the Correlation Function to Measure $H(z), D_{A}(z)$, and $\beta(z)$ from Sloan Digital Sky Survey Luminous Red Galaxies [arXiv:1205.5573 [astro-ph.CO]]

[20] X. Xu, A.J. Cuesta, N. Padmanabhan, D.J. Eisenstein and C.K. McBride, Measuring $D_{A}$ and $H$ at $z=0.35$ from the SDSS DR7 LRGs using baryon acoustic oscillations [arXiv:1206.6732 [astro-ph.CO]]

[21] C.-H. Chuang and Y. Wang, Measurements of $H(z)$ and $D_{A}(z)$ from the Two-Dimensional Two-Point Correlation Function of Sloan Digital Sky Survey Luminous Red Galaxies, Mon. Not. Roy. Astron. Soc. 426 (2012) 226 [arXiv:1102.2251 [astro-ph.CO]]

C.-H. Chuang and Y. Wang, Modeling the Anisotropic Two-Point Galaxy Correlation Function on Small Scales and Improved Measurements of $H(z), D_{A}(z)$, and $\beta(z)$ from the Sloan Digital Sky Survey DR7 Luminous Red Galaxies [arXiv:1209.0210 [astro-ph.CO]]

N.G. Busca et al [BOSS Collaboration], Baryon Acoustic Oscillations in the Ly- $\alpha$ forest of BOSS quasars, Astron. \&5 Astrophys. 552 (2013) A96 [arXiv:1211.2616 [astro-ph.CO]]

A. Slosar et al., Measurement of Baryon Acoustic Oscillations in the Lyman-alpha Forest Fluctuations in BOSS Data Release 9, [arXiv:1301.3459 [astro-ph.CO]]

[22] J. Miralda-Escude, Comment on the claimed radial BAO detection by Gaztanaga et al, [arXiv:0901.1219 [astro-ph]]

F. Sylos Labini, N.L. Vasilyev, Y.V. Baryshev and M. Lopez-Corredoira, Absence of anti-correlations and of baryon acoustic oscillations in the galaxy correlation function from the Sloan Digital Sky Survey DR7, Astron. ES Astrophys. 505 (2009) 981 [arXiv:0903.0950 [astro-ph.CO]] 
A. Cabre and E. Gaztañaga, Have Baryonic Acoustic Oscillations in the galaxy distribution really been measured?, Mon. Not. Roy. Astron. Soc. 412 (2011) L98 [arXiv:1011.2729 [astro-ph.CO]]

[23] R. Jimenez and A. Loeb, Constraining Cosmological Parameters Based on Relative Galaxy Ages, Astrophys. J. 573 (2002) 37 [arXiv:astro-ph/0106145]

S.M. Crawford, A.L. Ratsimbazafy, C.M. Cress, E.A. Olivier, S-L. Blyth and K.J. van der Heyden, Luminous Red Galaxies in Simulations: Cosmic Chronometers?, [arXiv:1004.2378 [astro-ph.CO]]

D.P. Carson and R.C. Nichol, The age-redshift relation for Luminous Red Galaxies in the Sloan Digital Sky Survey, Mon. Not. Roy. Astron. Soc. 408 (2010) 213 [arXiv:1006.2830 [astro-ph.CO]]

M. Moresco, R. Jimenez, A. Cimatti and L. Pozzetti, Constraining the expansion rate of the Universe using low-redshift ellipticals as cosmic chronometers, JCAP03(2011)045 [arXiv:1010.0831 [astro-ph.CO]]

T.J. Zhang and C. Ma, Constraints on the Dark Side of the Universe and Observational Hubble Parameter Data, Adv. Astron. 2010 (2010) 184284 [arXiv:1010.1307 [astro-ph.CO]]

M. Seikel, S. Yahya, R. Maartens and C. Clarkson, Using H(z) data as a probe of the concordance model, Phys. Rev. D86 (2012) 083001 [arXiv:1205.3431 [astro-ph.CO]]

[24] J. Simon, L. Verde and R. Jimenez, Constraints on the redshift dependence of the dark energy potential, Phys. Rev. D71 (2005) 123001 [arXiv:astro-ph/0412269]

D. Stern, R. Jimenez, L. Verde, M. Kamionkowski and S.A. Stanford, Cosmic Chronometers: Constraining the Equation of State of Dark Energy. I: H(z) Measurements, JCAP02(2010)008 [arXiv:0907.3149 [astro-ph.CO]]

[25] M. Moresco, A. Cimatti, R. Jimenez, L. Pozzetti, G. Zamorani, M. Bolzonella et al., Improved constraints on the expansion rate of the Universe up to z 1.1 from the spectroscopic evolution of cosmic chronometers, JCAP08(2012)006 [arXiv:1201.3609 [astro-ph.CO]]

[26] C. Zhang, H. Zhang, S. Yuan, T.-J. Zhang and Y.-C. Sun, Four New Observational H(z) Data From Luminous Red Galaxies Sloan Digital Sky Survey Data Release Seven, [arXiv:1207.4541 [astro-ph.CO]]

[27] T. Buchert, On average properties of inhomogeneous cosmologies, 2000, Proc. 9th JGRG conference, ed. Y. Eriguchi et al., p. 306 [arXiv:gr-qc/0001056]

[28] C. Wetterich, Can Structure Formation Influence the Cosmological Evolution?, Phys. Rev. D67 (2003) 043513 [arXiv:astro-ph/0111166]

[29] D.J. Schwarz, Accelerated expansion without dark energy [arXiv:astro-ph/0209584]

[30] S. Räsänen, Dark energy from backreaction, JCAP02(2004)003 [arXiv:astro-ph/0311257] S. Räsänen, Backreaction of linear perturbations and dark energy [arXiv:astro-ph/0407317]

[31] E.W. Kolb, S. Matarrese, A. Notari and A. Riotto, The effect of inhomogeneities on the expansion rate of the universe, Phys. Rev. D71 (2005) 023524 [arXiv:hep-ph/0409038]

[32] M.F. Shirokov and I.Z. Fisher, Isotropic Space with Discrete Gravitational-Field Sources. On the Theory of a Nonhomogeneous Universe, Astronomicheskii Zhurnal 39 (1962) 899, Reprinted in Sov. Astron. J. 6 (1963) 699, Reprinted in Gen. Rel. Grav. 30 (1998) 1411

[33] G.F.R. Ellis, Relativistic cosmology: its nature, aims and problems, 1984 The invited papers of the 10th international conference on general relativity and gravitation p. 215

G.F.R. Ellis and W. Stoeger, The 'fitting problem' in cosmology, Class. Quant. Grav. 4 (1987) 1697

[34] T. Buchert and J. Ehlers, Averaging inhomogeneous Newtonian cosmologies, Astron. ES Astrophys. 320 (1997) 1 [arXiv:astro-ph/9510056]

[35] T. Buchert, On average properties of inhomogeneous fluids in general relativity I: dust cosmologies, Gen. Rel. Grav. 32 (2000) 105 [arXiv:gr-qc/9906015]

[36] T. Buchert, On average properties of inhomogeneous fluids in general relativity II: perfect fluid cosmologies, Gen. Rel. Grav. 33 (2001) 1381 [arXiv:gr-qc/0102049]

[37] G.F.R. Ellis and T. Buchert, The universe seen at different scales, Phys. Lett. A347 (2005) 38 [arXiv:gr-qc/0506106]

[38] S. Räsänen, Backreaction as an alternative to dark energy and modified gravity [arXiv:1012.0784 [astro-ph.CO]] 
S. Räsänen, Backreaction: directions of progress, Class. Quant. Grav. 28 (2011) 164008 [arXiv:1102.0408 [astro-ph.CO]]

[39] C.H. Chuang, J.A. Gu and W.Y. Hwang, Inhomogeneity-Induced Cosmic Acceleration in a Dust Universe, Class. Quant. Grav. 25 (2008) 175001 [arXiv:astro-ph/0512651]

A. Paranjape and T.P. Singh, The Possibility of Cosmic Acceleration via Spatial Averaging in Lemaître-Tolman-Bondi Models, Class. Quant. Grav. 23 (2006) 6955 [arXiv:astro-ph/0605195] T. Kai, H. Kozaki, K.-i. Nakao, Y. Nambu and C.M. Yoo, Can inhomogeneities accelerate the cosmic volume expansion?, Prog. Theor. Phys. 117 (2007) 229 [arXiv:gr-qc/0605120]

A. Paranjape, The Averaging Problem in Cosmology, Ph.D. thesis, Tata Institute of Fundamental Research, Mumbai, 2009 [arXiv:0906.3165 [astro-ph.CO]]

[40] S. Räsänen, Cosmological acceleration from structure formation, Int. J. Mod. Phys. D15 (2006) 2141 [arXiv:astro-ph/0605632]

S. Räsänen, Accelerated expansion from structure formation, JCAP11(2006)003 [arXiv:astro-ph/0607626]

[41] S. Räsänen, Light propagation in statistically homogeneous and isotropic dust universes, JCAP02(2009)011 [arXiv:0812.2872 [astro-ph]]

[42] S. Räsänen, Light propagation in statistically homogeneous and isotropic universes with general matter content, JCAP03(2010)018 [arXiv:0912.3370 [astro-ph.CO]]

[43] M. Vonlanthen, S. Räsänen and R. Durrer, Model-independent cosmological constraints from the CMB, JCAP08(2010)023 [arXiv:1003.0810 [astro-ph.CO]]

[44] B. Audren, J. Lesgourgues, K. Benabed and S. Prunet, Conservative Constraints on Early Cosmology: an illustration of the Monte Python cosmological parameter inference code, JCAP02(2013)001 [arXiv:1210.7183 [astro-ph.CO]]

[45] S. Räsänen, Light propagation and the average expansion rate in near-FRW universes, Phys. Rev. D85 (2012) 083528 [arXiv:1107.1176 [astro-ph.CO]]

[46] S.R. Green and R.M. Wald, A new framework for analyzing the effects of small scale inhomogeneities in cosmology, Phys. Rev. D83 (2011) 084020 [arXiv:1011.4920 [gr-qc]]

S.R. Green and R.M. Wald, Examples of backreaction of small scale inhomogeneities in cosmology, Phys. Rev. D87 (2013) 124037 [arXiv:1304.2318 [gr-qc]]

[47] S. Räsänen, Evaluating backreaction with the peak model of structure formation, JCAP04(2008)026 [arXiv:0801.2692 [astro-ph]]

S. Räsänen, The effect of structure formation on the expansion of the universe, Int. J. Mod. Phys.

D17 (2008) 2543 [arXiv:0805.2670 [astro-ph]]

S. Räsänen, Structure formation as an alternative to dark energy and modified gravity, EAS

Publications Series (2009) $\mathbf{3 6} 63$ [arXiv:0811.2364 [astro-ph]]

[48] C. Clarkson, B.A. Bassett and T.C. Lu, A general test of the Copernican Principle, Phys. Rev. Lett. 101 (2008) 011301 [arXiv:0712.3457 [astro-ph]]

[49] J. Larena, J.-M. Alimi, T. Buchert, M. Kunz and P.-S. Corasaniti, Testing backreaction effects with observations, Phys. Rev. D79 (2009) 083011 [arXiv:0808.1161 [astro-ph]]

[50] S. Räsänen, Comment on "Nontrivial Geometries: Bounds on the Curvature of the Universe", Astropart. Phys. 30 (2008) 216 [arXiv:0705.2992 [astro-ph]]

[51] N. Suzuki et al. (The Supernova Cosmology Project), The Hubble Space Telescope Cluster Supernova Survey: V. Improving the Dark Energy Constraints Above $z_{\dot{z}} 1$ and Building an Early-Type-Hosted Supernova Sample, Astrophys. J. 746 (2012) 85 [arXiv:1105.3470 [astro-ph.CO]]

[52] P. Bull and T. Clifton, Local and non-local measures of acceleration in cosmology, Phys. Rev. D85 (2012) 103512 [arXiv:1203.4479 [astro-ph.CO]]

[53] D.W. Hogg et al., Cosmic homogeneity demonstrated with luminous red galaxies, Astrophys. J. 624 (2005) 54 [arXiv:astro-ph/0411197]

[54] M. Scrimgeour et al., The WiggleZ Dark Energy Survey: the transition to large-scale cosmic homogeneity, Mon. Not. Roy. Astron. Soc. 425 (2012) 116 [arXiv:1205.6812 [astro-ph.CO]] 
[55] S. Nadathur, Seeing patterns in noise: Gigaparsec-scale 'structures' that do not violate homogeneity [arXiv:1306.1700 [astro-ph.CO]]

[56] I.M.H. Etherington, On the definition of distance in general relativity, Philosophical Magazine 15 (1933) 761 Reprinted in Gen. Rel. Grav. 39 (2007) 1055

[57] G.F.R. Ellis, Relativistic Cosmology, 1971, General Relativity and Cosmology, ed. R.K. Sachs, Academic Press Inc., London, p. 104, Reprinted in Gen. Rel. Grav. 41 (2009) 581

[58] S. Nadathur and S. Sarkar, Reconciling the local void with the CMB, Phys. Rev. D83 (2011) 063506 [arXiv:1012.3460 [astro-ph.CO]]

[59] J.D. Hunter, Matplotlib: A 2D graphics environment, Computing In Science 6 Engineering 9 (2007) 90

[60] F. Ferrer and S. Räsänen, Dark energy and decompactification in string gas cosmology, JHEP02(2006)016 [arXiv:hep-th/0509225]

F. Ferrer, T. Multamäki and S. Räsänen, Fitting oscillating string gas cosmology to supernova data, JHEP04(2009)006 [arXiv:0812.4182 [hep-th]]

F. Ferrer, Cosmological acceleration from a gas of strings, Nucl. Phys. Proc. Suppl. 194 (2009) 218 [arXiv:0907.1342 [hep-th]]

[61] S. February, J. Larena, M. Smith and C. Clarkson, Rendering Dark Energy Void, Mon. Not. Roy. Astron. Soc. 405 (2010) 2231 [arXiv:0909.1479 [astro-ph.CO]]

[62] A.R. Neben and M.S. Turner, Beyond $H_{0}$ and $q_{0}$ : Cosmology is no longer just two numbers, Astrophys. J. 769 (2013) 133 [arXiv:1209.0480 [astro-ph.CO]]

[63] P.J.E. Peebles, Probing General Relativity on the Scales of Cosmology [arXiv:astro-ph/0410284]

[64] N. Jackson, The Hubble Constant, Living Reviews in Relativity 10 (2007) 4 [arXiv:0709.3924 [astro-ph]] G.A. Tammann, A. Sandage and B. Reindl, The expansion field: The value of $H_{0}$, Astron. E Astrophys. 15 (2008) 289 [arXiv:0806.3018 [astro-ph]]

A.G. Riess et al., A Redetermination of the Hubble Constant with the Hubble Space Telescope from a Differential Distance Ladder, Astrophys. J. 699 (2009) 539 [arXiv:0905.0695 [astro-ph.CO]]

[65] A.G. Riess et al., A 3\% Solution: Determination of the Hubble Constant with the Hubble Space Telescope and Wide Field Camera 3, Astrophys. J. 730 (2011) 119 [arXiv:1103.2976 [astro-ph.CO]]

[66] W.L. Freedman et al., Carnegie Hubble Program: A Mid-Infrared Calibration of the Hubble Constant, Astrophys. J. 758 (2012) 24 [arXiv:1208.3281 [astro-ph.CO]]

[67] P.M. Okouma, Y. Fantaye and B.A. Bassett, How Flat is Our Universe Really?, Phys. Lett. B719 (2013) 1 [arXiv:1207.3000 [astro-ph.CO]]

[68] C. Clarkson, T. Clifton, A. Coley and R. Sung, Observational Constraints on the Averaged Universe, Phys. Rev. D85 (2012) 043506 [arXiv:1111.2214 [astro-ph.CO]]

[69] P.A.R. Ade et al. [Planck Collaboration], Planck 2013 results. XVI. Cosmological parameters, [arXiv:1303.5076 [astro-ph.CO]]

[70] A. Shafieloo and C. Clarkson, Model independent tests of the standard cosmological model, Phys. Rev. D81 (2010) 083537 [arXiv:0911.4858 [astro-ph.CO]]

[71] E. Mörtsell and J. Jönsson, A model independent measure of the large scale curvature of the Universe [arXiv:1102.4485 [astro-ph.CO]]

[72] S. More, J. Bovy and D. W. Hogg, Cosmic transparency: A test with the baryon acoustic feature and type Ia supernovae, Astrophys. J. 696 (2009) 1727 [arXiv:0810.5553 [astro-ph]]

A. Avgoustidis, L. Verde and R. Jimenez, Consistency among distance measurements: transparency, BAO scale and accelerated expansion, JCAP06(2009)012 [arXiv:0902.2006 [astro-ph.CO]]

A. Avgoustidis, C. Burrage, J. Redondo, L. Verde and R. Jimenez, Constraints on cosmic opacity and beyond the standard model physics from cosmological distance measurements, JCAP10(2010)024 [arXiv:1004.2053 [astro-ph.CO]]

C. Escamilla-Rivera, R. Lazkoz, V. Salzano and I. Sendra, Tension between SN and BAO: current status and future forecasts, JCAP09(2011)003 [arXiv:1103.2386 [astro-ph.CO]]

A. Shafieloo, Crossing Statistic: Reconstructing the Expansion History of the Universe, JCAP08(2012)002 [arXiv:1204.1109 [astro-ph.CO]] 
S. Nesseris and J. Garcia-Bellido, A new perspective on Dark Energy modeling via Genetic Algorithms, JCAP11(2012)033 [arXiv:1205.0364 [astro-ph.CO]]

R. Nair, S. Jhingan and D. Jain, Cosmic distance duality and cosmic transparency, JCAP12(2012)028 [arXiv:1210.2642 [astro-ph.CO]]

J. Chen, P. Wu, H. Yu and Z. Li, Is the Cosmic Transparency Spatially Homogeneous?, JCAP10(2012)029 [arXiv:1210.2805 [gr-qc]]

[73] L.M. Krauss and B. Chaboyer, Age Estimates of Globular Clusters in the Milky Way: Constraints on Cosmology, Science 299 (2003) 65

[74] L. Verde, R. Jimenez and S. Feeney, The importance of local measurements for cosmology [arXiv:1303.5341 [astro-ph.CO]]

[75] S. Räsänen, Constraints on backreaction in dust universes, Class. Quant. Grav. 23 (2006) 1823 [arXiv:astro-ph/0504005]

[76] S. Das, A. Shafieloo and T. Souradeep, ISW effect as probe of features in the expansion history of the Universe [arXiv:1305.4530 [astro-ph.CO]]

[77] S. Räsänen, On the relation between the isotropy of the CMB and the geometry of the universe, Phys. Rev. D79 (2009) 123522 [arXiv:0903.3013 [astro-ph.CO]] 